\title{
COMPARISONS OF EQUIVALENCE RELATIONS ON OPEN PROJECTIONS
}

\author{
CHI-KEUNG NG AND NGAI-CHING WONG
}

\begin{abstract}
The aim of this article is to compare some equivalence relations among open projections of a $C^{*}$-algebra. Such equivalences are crucial in a decomposition scheme of $C^{*}$-algebras and is related to the Cuntz semigroups of $C^{*}$-algebras. In particular, we show that the spatial equivalence (as studied by H. Lin as well as by the authors) and the PZequivalence (as studied by C. Peligrad and L. Zsidó as well as by E. Ortega, M. Rørdam and $\mathrm{H}$. Thiel) are different, although they look very similar and conceptually the same.

In the development, we also show that the Murray-von Neumann equivalence and the Cuntz equivalence (as defined by Ortega, Rørdam and Thiel) coincide on open projections of $C_{0}(\Omega) \otimes \mathcal{K}\left(\ell^{2}\right)$ exactly when the canonical homomorphism from $\operatorname{Cu}\left(C_{0}(\Omega)\right)$ into $\operatorname{Lsc}\left(\Omega ; \overline{\mathbb{N}}_{0}\right)$ is bijective. Here, $\operatorname{Cu}\left(C_{0}(\Omega)\right)$ is the stabilized Cuntz semigroup, and $\operatorname{Lsc}\left(\Omega ; \overline{\mathbb{N}}_{0}\right)$ is the semigroup of lower semicontinuous functions from $\Omega$ into $\overline{\mathbb{N}}_{0}:=\{0,1,2, \ldots, \infty\}$.
\end{abstract}

\section{INTRODUCTION}

In their seminal works $[15]$ (see also $[9,14]$ ), Murray and von Neumann classified $W^{*}$ algebras into three types according to the abelianness and finiteness properties of their projections. The finiteness of projections is defined through an equivalence relation, the so-called Murray-von Neumann equivalence $\sim_{\text {Mv }}$. More precisely, for two projections $p$ and $q$ in a $W^{*}$-algebra $M$, we write $p \sim_{\mathrm{Mv}} q$ if there is a partial isometry $u$ in $M$ such that $p=u^{*} u$ and $q=u u^{*}$. A projection $p$ is finite in $M$ if $p$ is not equivalent to any of its proper subprojections. One can study a $W^{*}$-algebra by looking at the set of its projections, equipped with the order structure as well as the Murray-von Neumann equivalence.

In a similar fashion, in order to get a nice snapshot of a $C^{*}$-algebra, one studies its open projections under certain equivalence relations. For an example, classification frameworks for $C^{*}$-algebras were studied in [16] using either the so called "spatial equivalence" (denoted by $\sim_{\mathrm{sp}}$ ) as well as the "PZ-equivalence" (denoted by $\sim_{\mathrm{PZ}}$ ). Another example is that the PZ-equivalence was used in [18] to define what is called the "Cuntz equivalence" (denoted by $\sim_{\mathrm{Cu}}$ ), which is related to the Cuntz semigroups. These equivalences, however, are quite

Date: December 17, 2014.

2000 Mathematics Subject Classification. 46L05, 46L35.

Key words and phrases. $C^{*}$-algebra; open projection; equivalence relation; Cuntz semigroup.

The authors are supported by National Natural Science Foundation of China (11071126 and 11471168), and Taiwan MOST grant (102-2115-M-110-002-MY2). 
different from each other. In this paper, we are going to discuss some relationships among them.

Recall that if $A$ is a $C^{*}$-algebra, two open projections $p$ and $q$ of $A$ are "spatially equivalent" if there is a partial isometry in the bidual $A^{* *}$ of $A$ implementing a *-isomorphism between the corresponding hereditary $C^{*}$-subalgebras her $(p)$ and $\operatorname{her}(q)$. It is the same as the existence of a partial isometry that simultaneously implements the Murray-von Neumann equivalences of all the open subprojections of $p$ and those of $q$ (see [16, Proposition 2.7]). The spatial equivalence was studied by H. Lin in [12] as well as by the authors in [16] (and it was called the Cuntz equivalence in [12]).

On the other hand, $p$ and $q$ are "PZ-equivalent" if there is a partial isometry in $A^{* *}$ that implements an isomorphism between the corresponding right ideals $R(p)$ and $R(q)$ (see Proposition 3.1(a) below). The PZ-equivalence was first introduced by C. Peligrad and L. Zsidó in [19], and was studied in depth by E. Ortega, M. Rørdam and H. Thiel in [18]. In his book [11, 3.5.2], H. Lin also discussed a similar notion.

Since there is a canonical bijective correspondence between right ideals and hereditary $C^{*}$ subalgebras of a $C^{*}$-algebra, it is tempting to think that the PZ-equivalence is the same as the spatial equivalence. In fact, a statement before [18, Definition 3.9] seems to indicate this. In this article, we will show that, in contrast with the above expectation, the PZ-equivalence and the spatial equivalence are actually different, even for projections in a separable $C^{*}$-algebra of the form $C(\Omega) \otimes M_{2}$. We also give some interesting and hopefully useful comparisons of different equivalence relations on open projections.

This paper is organized as follows. After giving some preliminary and background results in Section 2, we give in Section 3 some equivalent formulations of the PZ-equivalence and those of the spatial equivalence, which show their similarities. Moreover, we find that one can determine the PZ-equivalence and the Cuntz equivalence (in the sense of [18]) of open projections of a $C^{*}$-algebra $A$ by looking at their atomic parts in the bidual $A^{* *}$ (Proposition $3.1(\mathrm{~b}))$.

In Section 4, we present an easier description for open projections in $C_{0}(\Omega) \otimes \mathcal{K}\left(\ell^{2}\right)$ (Proposition 4.1). We also give some interesting applications of this description in Corollaries 4.2 and 4.3 .

In Section 5, we compare equivalence relations on open projections of $C_{0}(\Omega) \otimes \mathcal{K}\left(\ell^{2}\right)$. In particular, we show that the PZ-equivalence is different from the spatial equivalence for projections in $C_{0}(\Omega) \otimes \mathcal{K}\left(\ell^{2}\right)$ (or even in $C_{0}(\Omega) \otimes M_{2}$; see Theorem 5.3). Moreover, we also show that two open projections of $C_{0}(\Omega) \otimes \mathcal{K}\left(\ell^{2}\right)$ are Murray-von Neumann equivalent as projections in $C_{0}(\Omega)^{* *} \bar{\otimes} \mathcal{B}\left(\ell^{2}\right)$ if and only if they produce the same element in the semigroup 
$\operatorname{Lsc}\left(\Omega ; \overline{\mathbb{N}}_{0}\right)$ of lower semicontinuous functions from $\Omega$ to the one-point compactification $\overline{\mathbb{N}}_{0}$ of $\mathbb{N} \cup\{0\}$ (Proposition 5.4). Consequently, the canonical homomorphism from the stabilized Cuntz semigroup $\operatorname{Cu}\left(C_{0}(\Omega)\right)$ to $\operatorname{Lsc}\left(\Omega ; \overline{\mathbb{N}}_{0}\right)$ is bijective if and only if the Cuntz equivalence coincides with the Murray-von Neumann equivalence. We also give some interesting consequences of this bijectivity (Corollaries 5.7 and 5.8).

\section{Preliminaries and Notations}

In this article, $A$ and $B$ are separable $C^{*}$-algebras, and $\mathcal{K}$ (respectively, $\mathcal{B}$ ) is the algebra of compact (respectively, bounded) linear operators on the separable infinite dimensional Hilbert space $\ell^{2}$. For any $\xi, \eta, \zeta \in \ell^{2}$, we set $\theta_{\xi, \eta}(\zeta):=\langle\zeta, \xi\rangle \eta$. We write SOT and WOT for, respectively, the strong operator topology and the weak operator topology on the space of bounded linear operators. For every $x \in A$ and any two subsets $M$ and $N$ of $A$, we put $M N:=\{b c: b \in M ; c \in N\}$ and $x M:=\{x\} M$.

Let $\left\{T_{i}\right\}_{i \in I}$ and $T$ be, respectively, a net and an element in the set, $\mathcal{B}(H ; K)$, of all bounded linear operators from a Hilbert space $H$ to another Hilbert space $K$. Recall that $\left\{T_{i}\right\}_{i \in I}$ is said to $S O T$ (respectively, WOT) converges to $T$ if $\left\|T_{i} \xi-T \xi\right\| \rightarrow 0$ for all $\xi \in H$ (respectively, $\left\langle T_{i} \xi-T \xi, \eta\right\rangle \rightarrow 0$ for all $\left.\xi, \eta \in H\right)$.

Throughout this paper, $\Omega$ is a second countable locally compact Hausdorff space. We use $\mathcal{O}(\Omega)$ to denote the set of all open subsets of $\Omega$. If $E \subseteq \Omega$, we denote by $\bar{E}$, Int $E$ and $\partial E$ the closure, the interior and the boundary of $E$ as a subset of $\Omega$. Moreover, $\chi_{E}$ is the indicator function of $E$ (i.e., $\chi_{E}=1$ on $E$ and vanishes elsewhere). For $U, V \in \mathcal{O}(\Omega)$, we write

$$
V \Subset U \text { if } \bar{V} \text { is compact and is contained in } U \text {. }
$$

Denote by $\mathbb{N}_{0}:=\mathbb{N} \cup\{0\}$, and consider $\overline{\mathbb{N}}_{0}:=\mathbb{N}_{0} \cup\{\infty\}$ (respectively, $\overline{\mathbb{N}}:=\mathbb{N} \cup\{\infty\}$ ) to be the one-point compactification of the discrete space $\mathbb{N}_{0}$ (respectively, $\mathbb{N}$ ).

Let $A^{* *}$ be the bidual of $A$ and let $\kappa_{A}: A \rightarrow A^{* *}$ be the canonical embedding. For simplicity, we will usually regard $A$ as a $C^{*}$-subalgebra of $A^{* *}$ without mentioning $\kappa_{A}$ if no confusion arises. We denote by $\sigma^{*}$ the weak-*-topology $\sigma\left(A^{* *}, A^{*}\right)$ on $A^{* *}$, and by $z_{\mathbf{a}} \in A^{* *}$ the central projection such that $z_{\mathbf{a}} A^{* *}$ is the atomic part of $A^{* *}$. Furthermore, $\operatorname{Proj}(A)$ is the set of projections in $A$, while $\operatorname{OP}(A) \subseteq \operatorname{Proj}\left(A^{* *}\right)$ is the set of open projections of $A$. Recall that a projection $p \in A^{* *}$ is open if there exists an increasing net $\left\{a_{i}\right\}_{i \in I}$ in $A_{+}$that $\sigma^{*}$-converges to $p$. For each $p \in \mathrm{OP}(A)$, the corresponding hereditary $C^{*}$-subalgebra, right ideal and linking algebra are given, respectively, by

$$
\operatorname{her}(p):=\{a \in A: p a p=a\}, \quad R(p):=\{a \in A: p a=a\}, \quad \text { and }
$$




$$
\mathfrak{L}(p):=\left(\begin{array}{cc}
\operatorname{her}(p) & R(p) \\
R(p)^{*} & A \operatorname{her}(p) A
\end{array}\right) .
$$

It is well-known that

$$
\begin{aligned}
\operatorname{her}(p) & =A \cap p A^{* *} p=R(p) R(p)^{*}=R(p) \cap R(p)^{*}, \\
R(p) & =A \cap p A^{* *}=\operatorname{her}(p) A=\left\{x \in A: x x^{*} \in \operatorname{her}(p)\right\},
\end{aligned}
$$

the $\sigma^{*}$-closure of her $(p)$ is $p A^{* *} p$, and the $\sigma^{*}$-closure of $R(p)$ is $p A^{* *}$. For every $a \in A_{+} \backslash\{0\}$, we consider $p_{a} \in \mathrm{OP}(A)$ to be the element defined by

$$
p_{a}:=\sigma^{*}-\lim _{n \rightarrow \infty}(a /\|a\|)^{1 / n} .
$$

It is easy to see that $\operatorname{her}\left(p_{a}\right)=\overline{a A a}$.

On the other hand, we say that $r \in \operatorname{Proj}\left(A^{* *}\right)$ is a closed projection of $A$ if $1-r \in \operatorname{OP}(A)$. A closed projection $r$ is said to be compact if there exists $a \in A_{+}$satisfying $r \leq a \leq 1$ (which implies $r a=r$ ). Clearly, if $A$ is unital, then any closed projection is compact. Let $p, q \in \mathrm{OP}(A)$. We use $\bar{p}$ to denote the smallest closed projection of $A$ dominating $p$. As in [18], we say that $p$ is compactly contained in $q$ (and is denoted by $p \Subset q$ ) if $\bar{p}$ is compact and $\bar{p} \leq q$. Now, we recall

- $p \sim_{\mathrm{Mv}} q$ if there is $u \in A^{* *}$ such that $p=u u^{*}$ and $q=u^{*} u$.

- $p \sim_{\mathrm{sp}} q$ if there is $u \in A^{* *}$ such that

$$
p=u u^{*}, q=u^{*} u, u^{*} \operatorname{her}(p) u=\operatorname{her}(q) \text {, and } u \operatorname{her}(q) u^{*}=\operatorname{her}(p) ;
$$

- $p \sim_{\mathrm{PZ}} q$ if there is $u \in A^{* *}$ such that

$$
p=u u^{*}, q=u^{*} u, u^{*} \operatorname{her}(p) \subseteq A, \text { and } u \operatorname{her}(q) \subseteq A ;
$$

- $p \sim_{\mathrm{Cu}} q$ if for any $r \Subset p$, there exists $s \Subset q$ with $r \sim_{\mathrm{PZ}} s$ and vice versa;

If it happens that $p, q \in A$, then we write

- $p \sim_{A} q$ if there is $u \in A$ such that $p=u u^{*}$ and $q=u^{*} u$.

Let us state the following known facts for open projections $p, q$ of $A$ (see e.g. [18] and [16]): (F1) $p \sim_{\mathrm{PZ}} q \Rightarrow p \sim_{\mathrm{sp}} q \Rightarrow p \sim_{\mathrm{Mv}} q$ (in fact, the element $u$ satisfying the requirement for $\sim_{\mathrm{PZ}}$ will satisfy the requirement for $\sim_{\mathrm{sp}}$ ).

(F2) $p \sim_{\mathrm{PZ}} q \Rightarrow p \sim_{\mathrm{Cu}} q \Rightarrow p \sim_{\mathrm{Mv}} q$.

(F3) $p \sim_{\mathrm{Mv}} q \Rightarrow p$ and $q$ have the same central support, i.e., $A \operatorname{her}(p) A=A \operatorname{her}(q) A$.

(F4) $p \sim_{\mathrm{sp}} q \Leftrightarrow$ there exists a partial isometry $v \in A^{* *}$ satisfying

$$
\operatorname{her}(p) \subseteq v \operatorname{her}(q) v^{*} \text { and } \operatorname{her}(q) \subseteq v^{*} \operatorname{her}(p) v .
$$

(F5) $p \in A$ and $p \sim_{\mathrm{PZ}} q \Rightarrow q \in A$ and $p \sim_{A} q$ (notice that $u^{*}=u^{*} p \in A$ ).

(F6) $p, q \in A$ and $p \sim_{A} q \Rightarrow p \sim_{\mathrm{PZ}} q$. 
Finally, suppose that $B$ is a hereditary $C^{*}$-subalgebra of $A$ and $p, q \in \operatorname{Proj}\left(B^{* *}\right)$. Then we have the following facts (see e.g. [16]).

(H1) $p \in \mathrm{OP}(B)$ if and only if its canonical images in $\operatorname{Proj}\left(A^{* *}\right)$ lies in $\mathrm{OP}(A)$.

(H2) If $p \in \mathrm{OP}(B)$, then $A \cap p A^{* *} p=B \cap p B^{* *} p$.

(H3) If $p, q \in \mathrm{OP}(B)$, then $p \sim_{\mathrm{sp}} q$ as elements in $\mathrm{OP}(A)$ if and only if $p \sim_{\mathrm{sp}} q$ as elements in $\mathrm{OP}(B)$.

\section{REFORMUlation OF $\sim_{\mathrm{PZ}}$ AND $\sim_{\mathrm{sp}}$}

By a result of H. Lin (see [10, Theorem 9]), one knows that there is a projection $p$ in a separable simple unital $C^{*}$-algebra $A$ such that $p \sim_{\mathrm{Mv}} 1$ but $p \nsim_{\mathrm{sp}} 1$. In fact, from the proof of Theorem 9 in [10], there exists $u \in A$ such that $u u^{*}=1, u^{*} u \leq p$ and $p A p$ is not isomorphic to $A$. As $p \leq 1$ as well, by a well-known fact in von Neumann algebra, one has $p \sim_{\mathrm{Mv}} 1$ (note that, the partial isometry is in $A^{* *}$ instead of $A$ ). On the other hand, since $p A p \not A$, one has $p \nsim_{\mathrm{sp}} 1$.

One of the concerns in this paper is to determine whether $\sim_{\mathrm{PZ}}$ coincides with $\sim_{\mathrm{sp}}$. Let us first begin with the following easy reformulation of $\sim_{\mathrm{PZ}}$. Part (a) of which can be regarded as an analogue of (F4) for $\sim_{\mathrm{PZ}}$. In view of this result as well as the bijective correspondence between right ideals and hereditary $C^{*}$-subalgebras, it seems plausible that the equivalence relations $\sim_{\mathrm{PZ}}$ and $\sim_{\mathrm{sp}}$ might be the same. Nevertheless, we will see in Theorem 5.3 below that it is not the case.

Proposition 3.1. Let $A$ be a $C^{*}$-algebra and let $p, q \in \mathrm{OP}(A)$. For any $a \in A$, we set $\kappa_{A}^{\mathbf{a}}(a):=z_{\mathbf{a}} \kappa_{A}(a)$ (i.e. the atomic part of $\kappa_{A}(a)$ in $A^{* *}$ ).

(a) The following statements are equivalent.

(1) $p \sim_{\mathrm{PZ}} q$

(2) There is a partial isometry $v \in A^{* *}$ with $R(p)=v R(q)$ and $R(q)=v^{*} R(p)$.

(3) There is a partial isometry $v \in A^{* *}$ with $\operatorname{her}(p) \subseteq v R(q)$ and $\operatorname{her}(q) \subseteq v^{*} R(p)$.

(4) There is a partial isometry $v \in A^{* *}$ with

$$
\left(v^{*} \oplus 1\right) \mathfrak{L}(p)(v \oplus 1)=\mathfrak{L}(q) \text { and }(v \oplus 1) \mathfrak{L}(q)\left(v^{*} \oplus 1\right)=\mathfrak{L}(p) .
$$

(b) If $A$ is separable, then $p \sim_{\mathrm{PZ}} q$ if and only if there exists $w \in z_{\mathbf{a}} A^{* *}$ satisfying

$$
w w^{*}=z_{\mathbf{a}} p, w^{*} w=z_{\mathbf{a}} q, w^{*} \kappa_{A}^{\mathbf{a}}(\operatorname{her}(p)) \subseteq \kappa_{A}^{\mathbf{a}}(A) \quad \text { and } \quad w \kappa_{A}^{\mathbf{a}}(\operatorname{her}(q)) \subseteq \kappa_{A}^{\mathbf{a}}(A) .
$$


Proof: (a) Clearly one has (2) $\Rightarrow(3)$ as well as (2) $\Leftrightarrow(4)$ (see (F1) and (F3)).

$(1) \Rightarrow(2)$. Let $v \in A^{* *}$ be a partial isometry satisfying the requirement for $p \sim_{\mathrm{PZ}} q$. Then $v^{*} R(p) \subseteq A \cap q A^{* *}=R(q)$ as well as $v R(q) \subseteq R(p)$. The two equalities follows from $p=v v^{*}$ and $q=v^{*} v$.

$(3) \Rightarrow(1)$. By Statement (3), one has her $(p) \subseteq v R(q)(v R(q))^{*}=v \operatorname{her}(q) v^{*}$ and $p \leq v v^{*}$. If we set $w:=p v$, then $p=w w^{*}, R(p) \subseteq w R(q)$ and $R(q) \subseteq w^{*} R(p)$. Furthermore, if $u:=w q$, then $q=u^{*} u$ (as her $\left.(q) \subseteq v^{*} \operatorname{her}(p) v\right)$ and $u u^{*}=w q w^{*}=p v q v^{*} p \leq p$. On the other hand, since $\operatorname{her}(p) \subseteq w \operatorname{her}(q) w^{*}$, we see that $p \leq w q w^{*}$ and hence $p=u u^{*}$. Moreover, we have $u^{*} \operatorname{her}(p) \subseteq u^{*} R(p)=R(q) \subseteq A$ and $u \operatorname{her}(q) \subseteq A$.

(b) Let $Q_{\mathbf{a}}: A^{* *} \rightarrow A^{* *}$ be given by $Q_{\mathbf{a}}(x):=z_{\mathbf{a}} x$. By [20, Corollary 4.5.13], $Q_{\mathbf{a}}$ is injective on both $\operatorname{OP}(A)$ and $\kappa_{A}(A)$. As $A$ is separable, one can find $a \in \operatorname{her}(p)_{+}$with $\|a\|=1$ and $p=p_{a}$. Suppose that $w \in z_{\mathbf{a}} A^{* *}$ satisfies the conditions in the statement. It is not hard to see that $w^{*} \kappa_{A}^{\mathbf{a}}(\operatorname{her}(p)) w=\kappa_{A}^{\mathbf{a}}(\operatorname{her}(q))$ and there exist unique elements $b \in A_{+}$and $x \in A$ with $\kappa_{A}^{\mathbf{a}}(b)=w^{*} \kappa_{A}^{\mathbf{a}}(a) w \in \kappa_{A}^{\mathbf{a}}(A)$ and $\kappa_{A}^{\mathbf{a}}(x)=w^{*} \kappa_{A}^{\mathbf{a}}(a)^{1 / 2} \in \kappa_{A}^{\mathbf{a}}(A)$. Moreover, as $p_{b}=\sigma^{*}-\lim _{n} \kappa_{A}(b)^{1 / n}$, we have

$$
Q_{\mathbf{a}}\left(p_{b}\right)=\sigma^{*}-\lim _{n} \kappa_{A}^{\mathbf{a}}(b)^{1 / n}=\sigma^{*}-\lim _{n}\left(w^{*} \kappa_{A}^{\mathbf{a}}(a) w\right)^{1 / n}=w^{*} Q_{\mathbf{a}}\left(p_{a}\right) w=Q_{\mathbf{a}}(q)
$$

and hence $q=p_{b}$. Furthermore, since $\kappa_{A}^{\mathbf{a}}\left(x x^{*}\right)=\kappa_{A}^{\mathbf{a}}(b)$ and $\kappa_{A}^{\mathbf{a}}\left(x^{*} x\right)=\kappa_{A}^{\mathbf{a}}(a)^{1 / 2} z_{\mathbf{a}} p \kappa_{A}^{\mathbf{a}}(a)^{1 / 2}=$ $\kappa_{A}^{\mathrm{a}}(a)$, we know that $a=x^{*} x$ and $b=x x^{*}$. Thus, $p=p_{a} \sim_{\mathrm{Pz}} p_{b}=q$ by [18, Proposition 4.3]. The converse is obvious.

Elements in $\mathrm{OP}^{\mathbf{a}}(A):=z_{\mathbf{a}} \mathrm{OP}(A)$ are first studied in [2], and are called $q$-open projections of $A$. In fact, a projection $p_{1} \in z_{\mathbf{a}} A^{* *}$ is said to be $q$-open if there is a hereditary $C^{*}$ subalgebra $B \subseteq A$ such that the $\sigma\left(z_{\mathbf{a}} A^{* *}, z_{\mathbf{a}} A^{*}\right)$-closure of $\kappa_{A}^{\mathbf{a}}(B)$ equals $p_{1} A^{* *}$ (see [2]), and it is not hard to check that it is equivalent to $p_{1} \in \mathrm{OP}^{\mathrm{a}}(A)$.

Suppose that $A$ is separable. It is easy to see that if one replaces $A^{* *}, R(p)$ and $R(q)$ in Statements (2), (3) and (4) in Proposition 3.1(a) above with $z_{\mathbf{a}} A^{* *}, z_{\mathbf{a}} R(p)$ and $z_{\mathbf{a}} R(q)$, respectively, then the resulting statements are also equivalent to $p \sim_{\mathrm{PZ}} q$. Furthermore, by [1, Theorem II.17] (see also [20, Corollary 4.5.13]), if $r$ (respectively, $s$ ) is either an open or a closed projection of $A$, then $r \leq s$ if and only if $z_{\mathbf{a}} r \leq z_{\mathbf{a}} s$. In particular, if $p_{1}, q_{1} \in \operatorname{OP}^{\mathbf{a}}(A)$, we say that $p_{1}$ is $q$-compactly contained in $q_{1}$ if there exist $r_{1} \in \mathrm{OP}^{\mathbf{a}}(A)$ and $a \in A_{+}$such that $r_{1} p_{1}=0$ and $z_{\mathbf{a}}-r_{1} \leq \kappa_{A}^{\mathbf{a}}(a) \leq q_{1}$. Clearly, if $p, q \in \mathrm{OP}(A)$, then $z_{\mathbf{a}} p$ is $q$-compactly contained in $z_{\mathbf{a}} q$ if and only if $p$ is compactly contained in $q$. This tells us that one can determine $\sim_{\mathrm{PZ}}$ and $\sim_{\mathrm{Cu}}$ by looking at the images of $A$ and $\mathrm{OP}(A)$ in $z_{\mathbf{a}} A^{* *}$. 
We do not know whether the corresponding statements of Proposition 3.1(b) for $\sim_{\mathrm{Mv}}$ and $\sim_{\text {sp }}$ hold. Note that in the case when $A=C_{0}(\Omega) \otimes \mathcal{K}$, the corresponding statement for $\sim_{\mathrm{Mv}}$ is precisely Proposition 5.4, while the lack of the corresponding statement for $\sim_{\mathrm{sp}}$ is one of the difficulty in proving Theorem 5.3.

In the following, we give some reformulations of $\sim_{\mathrm{sp}}$, which are the analogues of both Proposition 3.1(a) and [18, Proposition 3.3]. Let us first set some notations. Let $\tilde{A}$ be the subalgebra of the multiplier algebra $M(A)$ generated by $A$ and 1 . We put

$$
\mathfrak{H}_{A}:=\left\{x \in A^{* *}: x \tilde{A} x^{*} \subseteq A, x^{*} \tilde{A} x \subseteq A\right\} .
$$

It is easy to see that

$$
\mathfrak{H}_{A}=\left\{x \in A^{* *}: x A x^{*} \subseteq A, x^{*} A x \subseteq A\right\} \cap\left\{x \in A^{* *}: x x^{*} \in A, x^{*} x \in A\right\} .
$$

Clearly, when $A$ is unital, $\mathfrak{H}_{A}$ coincides with the first set on the right hand side of the above equation. However, it can be shown by examples that neither of the two sets on the right hand side equals $\mathfrak{H}_{A}$. Furthermore, if $A$ is the unital $C^{*}$-algebra of convergent complex sequences (identified with $c_{0} \oplus \mathbb{C}$ ), then $\mathfrak{H}_{A}=\left\{\left(\left(\alpha_{n}\right)_{n \in \mathbb{N}}, \alpha_{\infty}\right) \in \ell^{\infty} \oplus \mathbb{C}:\left|\alpha_{n}\right| \rightarrow\left|\alpha_{\infty}\right|\right\}$, which is not a vector subspace of $A^{* *}$. The reason for the introduction of the set $\mathfrak{H}_{A}$ is its relation with $\sim_{\mathrm{sp}}$ as stated in the following proposition. Part (a) of this proposition and its argument explain the difference between $\sim_{\mathrm{PZ}}$ and $\sim_{\mathrm{sp}}$ : the right ideal corresponding to $u \operatorname{her}(q) u^{*}$ is $A \cap u \check{R}(q)$ instead of $u R(q)$. However, one cannot use it to conclude that $\sim_{\mathrm{PZ}}$ and $\sim_{\mathrm{sp}}$ are different since the choice for $u$ is not unique.

Proposition 3.2. Let $A$ be a $C^{*}$-algebra and $p, q \in \mathrm{OP}(A)$. Set

$$
\check{R}(p):=\left\{x \in A^{* *}: x \tilde{A} x^{*} \in \operatorname{her}(p)\right\} .
$$

(a) $p \sim_{\mathrm{sp}} q$ if and only if there is a partial isometry $v \in A^{* *}$ such that $\check{R}(p)=v \check{R}(q)$ and $\check{R}(q)=v^{*} \check{R}(p)$, which is equivalent to $\operatorname{her}(p) \subseteq v \operatorname{her}(q) \mathfrak{H}_{A}$ and $\operatorname{her}(q) \subseteq v^{*} \operatorname{her}(p) \mathfrak{H}_{A}$.

(b) If $x \in \mathfrak{H}_{A}$, then $p_{x^{*} x} \sim_{\mathrm{sp}} p_{x x^{*}}$.

(c) If $p \sim_{\mathrm{sp}} q$ and $p=p_{a}$ for some $a \in A_{+}$, there is $x \in \mathfrak{H}_{A}$ such that $a=x^{*} x$ and $q=p_{x x^{*}}$.

Proof: (a) Suppose that $u \in A^{* *}$ is a partial isometry satisfying the requirement for $p \sim_{\mathrm{sp}} q$. For any $y \in \check{R}(q)$ and $b \in \tilde{A}$, one has $y b y^{*} \in \operatorname{her}(q)=u^{*} \operatorname{her}(p) u$, and so $u y \in \check{R}(p)$ and $y=u^{*} u y \in u^{*} \check{R}(p)$ (observe that $y \in q A^{* *}$ as $y y^{*} \in \operatorname{her}(q)$ ). Similarly, $\check{R}(p) \subseteq u \check{R}(q)$, and hence $\check{R}(p)=u \check{R}(q)$ as well as $\check{R}(q)=u^{*} \check{R}(p)$. Conversely, suppose that $v \in A^{* *}$ satisfying $\check{R}(p) \subseteq v \check{R}(q)$ and $\check{R}(q) \subseteq v^{*} \check{R}(p)$. For each $x \in \operatorname{her}(p)_{+} \subseteq \check{R}(p)$, there exists $y \in \check{R}(q)$ with $x^{1 / 2}=v y$, and hence $x=v y y^{*} v^{*} \in v \operatorname{her}(q) v^{*}$. Consequently, $\operatorname{her}(p) \subseteq v \operatorname{her}(q) v^{*}$. Similarly, $\operatorname{her}(q) \subseteq v^{*} \operatorname{her}(p) v$ and $(\mathrm{F} 4)$ gives $p \sim_{\mathrm{sp}} q$. 
Next, we show that $p \sim_{\mathrm{sp}} q$ implies the two inclusions in the second half of the statement. In fact, if $u$ is as above, then $\operatorname{her}(p)=\operatorname{her}(p) \operatorname{her}(p) \subseteq u \operatorname{her}(q) \operatorname{her}(q) u^{*} \operatorname{her}(p) \subseteq u \operatorname{her}(q) \mathfrak{H}_{A}$ (it is easy to see that $\operatorname{her}(q) u^{*} \operatorname{her}(p) \subseteq \mathfrak{H}_{A}$ ). Similarly, $\operatorname{her}(q) \subseteq u^{*} \operatorname{her}(p) \mathfrak{H}_{A}$. Conversely, if $v \in A^{* *}$ is a partial isometry with $\operatorname{her}(p) \subseteq v \operatorname{her}(q) \mathfrak{H}_{A}$ and $\operatorname{her}(q) \subseteq v^{*} \operatorname{her}(p) \mathfrak{H}_{A}$, then the argument above tells us that $p \sim_{\mathrm{sp}} q$ (since her $(q) \mathfrak{H}_{A} \subseteq \check{R}(q)$ ).

(b) Let $a:=x^{*} x$ and $b:=x x^{*}$. Suppose that $x=u a^{1 / 2}$ is the polar decomposition of $x$ in $A^{* *}$. As $p_{a}=u^{*} u$ and $p_{b}=u u^{*}$, one has

$$
\begin{aligned}
u \operatorname{her}\left(p_{a}\right) u^{*}=u \overline{a^{3 / 2} A a^{3 / 2}} u^{*}=\overline{x x^{*} x A x^{*} x x^{*}} & \subseteq \overline{x x^{*} A x x^{*}}=\operatorname{her}\left(p_{b}\right) \\
& \subseteq \overline{x A x^{*}}=u \overline{a^{1 / 2} A a^{1 / 2}} u^{*}=u \operatorname{her}\left(p_{a}\right) u^{*} .
\end{aligned}
$$

Similarly, $u^{*} \operatorname{her}\left(p_{b}\right) u=\operatorname{her}\left(p_{a}\right)$.

(c) Without loss of generality, we may assume that $\|a\|=1$ (notice that if $p=0$, then so is $q$ ). Suppose that $u \in A^{* *}$ is a partial isometry satisfying the requirement for $p \sim_{\mathrm{sp}} q$. Set $b:=u^{*} a u \in \operatorname{her}(q)$ and $x:=u^{*} a^{1 / 2}$. Then $x^{*} x=a \in A, x x^{*}=b \in A$ and $p_{b}=q$ (as in [18, Proposition 3.3]). Moreover, $x A x^{*}=u^{*} a^{1 / 2} A a^{1 / 2} u \subseteq \operatorname{her}(q) \subseteq A$. Finally, as $u x=a^{1 / 2}=u b^{1 / 2} u^{*}$, we have $x=b^{1 / 2} u^{*}$ and $x^{*} A x=u b^{1 / 2} A b^{1 / 2} u^{*} \subseteq u$ her $(q) u^{*} \subseteq A$. Now, (3.1) tells us that $x \in \mathfrak{H}_{A}$.

\section{4. q-OPEN AND $q$-COMPACT PROJECTIONS OF $C_{0}(\Omega) \otimes \mathcal{K}$}

In this section, we look at the case when $A=C_{0}(\Omega) \otimes \mathcal{K}$. Note that $z_{\mathbf{a}} A^{* *} \cong \ell^{\infty}(\Omega ; \mathcal{B})$. For a projection $q$ in $A^{* *}$, we write $q^{\mathbf{a}}:=z_{\mathbf{a}} q$ for its atomic part. Then $q^{\mathbf{a}}(\omega) \in \operatorname{Proj}(\mathcal{B})$, for any $\omega \in \Omega$. Denote by

$$
\Gamma_{k}^{q}:=\left\{\omega \in \Omega: \operatorname{rank} q^{\mathbf{a}}(\omega)=k\right\} \quad\left(k \in \overline{\mathbb{N}}_{0}\right) .
$$

Let us first describe the set

$$
\mathrm{OP}^{\mathbf{a}}(A):=\left\{p^{\mathbf{a}}: p \in \mathrm{OP}(A)\right\} \subseteq \ell^{\infty}(\Omega ; \mathcal{B})
$$

of $q$-open projections. This description could be known to experts but since it is not stated explicitly anywhere, we present its details here. Notice that the only place in this section where the second countability of $\Omega$ is needed is to apply the materials in $[4, \S 5$.G]. Thus, [4, 5.20] seems to indicate that the second countable assumption on $\Omega$ can be dropped for the results in this section. 
Proposition 4.1. Let $A=C_{0}(\Omega) \otimes \mathcal{K}$. For any $h: \Omega \rightarrow \operatorname{Proj}(\mathcal{B})$, one has $h \in \mathrm{OP}^{\mathbf{a}}(A)$ if and only if for any $\omega_{\infty} \in \Omega$, the map $\omega \mapsto h(\omega) h\left(\omega_{\infty}\right)$ is continuous at $\omega_{\infty}$ with respect to SOT on $\mathcal{B}$.

Proof: $\Rightarrow)$. If $p \in \mathrm{OP}(A)$ and $\left\{a_{i}\right\}_{i \in I}$ is an increasing net in $A_{+}$that $\sigma^{*}$-converges to $p$, then $\left\langle a_{i}(\omega) \xi, \xi\right\rangle \uparrow\left\langle p^{\mathbf{a}}(\omega) \xi, \xi\right\rangle$ for any $\omega \in \Omega$ and $\xi \in \ell^{2}$. This shows that the map $\omega \mapsto\left\langle p^{\mathbf{a}}(\omega) \xi, \xi\right\rangle$ is lower semicontinuous. Hence, if $\left\{\omega_{j}\right\}_{j \in J}$ is a net in $\Omega$ converging to $\omega_{\infty}$, we have

$$
\left\|p^{\mathbf{a}}\left(\omega_{\infty}\right) \xi\right\|^{2} \leq \liminf \left\|p^{\mathbf{a}}\left(\omega_{j}\right) \xi\right\|^{2} \quad\left(\xi \in \ell^{2}\right) .
$$

Consequently, for any $\zeta \in p^{\mathbf{a}}\left(\omega_{\infty}\right)\left(\ell^{2}\right)$ and $\epsilon>0$, there exists $j_{0} \in J$ with

$$
\|\zeta\|^{2} \leq\left\|p^{\mathbf{a}}\left(\omega_{j}\right) \zeta\right\|^{2}+\epsilon \quad\left(j \geq j_{0}\right)
$$

i.e. $\left\|\zeta-p^{\mathbf{a}}\left(\omega_{j}\right) \zeta\right\|^{2}=\|\zeta\|^{2}-\left\|p^{\mathbf{a}}\left(\omega_{j}\right) \zeta\right\|^{2} \rightarrow 0$. In other words,

$$
p^{\mathbf{a}}\left(\omega_{j}\right) p^{\mathbf{a}}\left(\omega_{\infty}\right) \stackrel{S O T}{\longrightarrow} p^{\mathbf{a}}\left(\omega_{\infty}\right)
$$

$\Leftarrow$ ). It suffices to verify that $h$ is strongly lsc in the sense of $[4, \S 5$.G] (see the discussion in [4, p. 980]). In other words, we are required to show that $h$ satisfies Conditions (i), (ii) and (iii) in $[4,5.19]$. Notice that the first two conditions automatically hold because $h(\Omega) \subseteq \operatorname{Proj}(\mathcal{B})$. Moreover, by the argument of $[4,5.19]$, subjected to our projection-valued function $h$, we only need to show the following weaker version of Condition (iii):

For any $\epsilon>0$ and $K \in \mathcal{K}$ with $0 \leq K \leq h\left(\omega_{\infty}\right)$, there is a neighbourhood $U$ of $\omega_{\infty}$ satisfying

$$
K \leq h(\omega)+\epsilon \quad(\omega \in U) .
$$

Now, assume that $0 \leq K \leq h\left(\omega_{\infty}\right)$. One can find an orthonormal subset $\left\{\eta_{k}\right\}_{k \in \mathbb{N}}$ in the range of the projection $h\left(\omega_{\infty}\right)$ such that $K=\sum_{k=1}^{\infty} \lambda_{k} \theta_{\eta_{k}, \eta_{k}}$ for a null sequence $\left\{\lambda_{k}\right\}_{k \in \mathbb{N}}$ of positive real numbers. Since $\lambda_{k} \rightarrow 0$, there is $N \in \mathbb{N}$ with $0 \leq \lambda_{k}<\epsilon / 2$ whenever $k \geq N$. By the hypothesis, we have a neighbourhood $U$ of $\omega_{\infty}$ such that

$$
\left\|h(\omega) \eta_{k}-\eta_{k}\right\|<\frac{\epsilon}{8 N} \quad(\omega \in U ; k=1,2, \ldots, N) .
$$

Thus, for any $\xi \in \ell^{2}$ and $\omega \in U$, we have

$$
\begin{aligned}
& \left\langle\sum_{k=1}^{N} \theta_{\eta_{k}, \eta_{k}} \xi, \xi\right\rangle \\
\leq & \sum_{k=1}^{N}\left(\left|\left\langle\xi, h(\omega) \eta_{k}\right\rangle\right|+\left|\left\langle\xi,\left(\eta_{k}-h(\omega) \eta_{k}\right)\right\rangle\right|\right)^{2} \\
\leq & \left\langle h(\omega)\left(\sum_{k=1}^{N} \theta_{\eta_{k}, \eta_{k}}\right) h(\omega) \xi, \xi\right\rangle+\sum_{k=1}^{N} 2\|\xi\|^{2}\left\|h(\omega) \eta_{k}-\eta_{k}\right\|+\|\xi\|^{2}\left\|h(\omega) \eta_{k}-\eta_{k}\right\|^{2} \\
\leq & \langle h(\omega) \xi, \xi\rangle+\frac{\epsilon}{2}\|\xi\|^{2} .
\end{aligned}
$$


Finally, as $\lambda_{k} \leq 1(k \in \mathbb{N})$, we have

$$
K-\sum_{k=N+1}^{\infty} \lambda_{k} \theta_{\eta_{k}, \eta_{k}} \leq \sum_{k=1}^{N} \theta_{\eta_{k}, \eta_{k}} \leq h(\omega)+\frac{\epsilon}{2}
$$

and (4.2) holds (since $\left\{\eta_{k}\right\}_{k \in \mathbb{N}}$ is an orthonormal set and $\lambda_{k}<\epsilon / 2$ for $k \geq N$ ).

Let $p \in \mathrm{OP}\left(C_{0}(\Omega) \otimes \mathcal{K}\right)$, and let $\left\{\omega_{j}\right\}_{j \in J}$ be a net in $\Omega$ that converges to $\omega_{\infty} \in \Omega$.

(O1) If $\operatorname{rank} p^{\mathbf{a}}\left(\omega_{\infty}\right)=\infty$, then $\operatorname{rank} p^{\mathbf{a}}\left(\omega_{j}\right) \rightarrow \infty$. On the other hand, if $\operatorname{rank} p^{\mathbf{a}}\left(\omega_{\infty}\right)<\infty$, then $\operatorname{rank} p^{\mathbf{a}}\left(\omega_{\infty}\right) \leq \operatorname{rank} p^{\mathbf{a}}\left(\omega_{j}\right)$ eventually.

Indeed, assume on the contrary that there exist $k \in \mathbb{N}$ and a subsequence $\left\{\omega_{j_{n}}\right\}_{n \in \mathbb{N}}$ satisfying $\operatorname{rank} p^{\mathbf{a}}\left(\omega_{j_{n}}\right) \leq k<\operatorname{rank} p^{\mathbf{a}}\left(\omega_{\infty}\right)(n \in \mathbb{N})$. If $\left\{\xi_{1}, \ldots, \xi_{k+1}\right\}$ is an orthonormal subset in $p^{\mathbf{a}}\left(\omega_{\infty}\right)\left(\ell^{2}\right)$, then Relation (4.1) gives $j_{0} \in J$ such that $\left\langle p^{\mathbf{a}}\left(\omega_{j}\right) \xi_{i}, \xi_{i}\right\rangle \geq 1-$ $(2 k+2)^{-1}$ for any $i=1, \ldots, k+1$ and $j \geq j_{0}$. By extending $\left\{\xi_{1}, \ldots, \xi_{k+1}\right\}$ to an orthonormal basis of $\ell^{2}$, we arrive at the contradiction that $\operatorname{rank} p^{\mathbf{a}}\left(\omega_{j_{n}}\right) \geq k+1 / 2$ when $n$ is so large that $j_{n} \geq j_{0}$.

(O2) $\bigcup_{k \geq n} \Gamma_{k}^{p} \cup \Gamma_{\infty}^{p} \in \mathcal{O}(\Omega)$ for any $n \in \mathbb{N}$ because of (O1).

(O3) $\left(1-p^{\mathbf{a}}\right)\left(\omega_{j}\right)-\left(1-p^{\mathbf{a}}\right)\left(\omega_{j}\right)\left(1-p^{\mathbf{a}}\right)\left(\omega_{\infty}\right) \stackrel{\text { SOT }}{\longrightarrow} 0$ by Proposition 4.1. This property is actually equivalent to $1-p^{\mathbf{a}}$ being $q$-closed.

Corollary 4.2. Let $r$ be a compact projection of $A=C_{0}(\Omega) \otimes \mathcal{K}$. Suppose that $\left\{\omega_{j}\right\}_{j \in J}$ is a net in $\Omega$ converging to $\omega_{\infty} \in \Omega$.

(a) $\left\|r^{\mathbf{a}}\left(\omega_{j}\right)-r^{\mathbf{a}}\left(\omega_{j}\right) r^{\mathbf{a}}\left(\omega_{\infty}\right)\right\| \rightarrow 0$.

(b) $\operatorname{rank} r^{\mathbf{a}}\left(\omega_{j}\right) \leq \operatorname{rank} r^{\mathbf{a}}\left(\omega_{\infty}\right)$ for large enough $j$.

(c) $\operatorname{rank} r^{\mathbf{a}}(\omega)<\infty(\omega \in \Omega)$ and $\bigcup_{k \geq n} \Gamma_{k}^{r}$ is a compact subset of $\Omega(n \in \mathbb{N})$.

(d) There is $n_{0} \in \mathbb{N}$ such that $\operatorname{rank} r(\omega) \leq n_{0}(\omega \in \Omega)$.

(e) $\left.r^{\mathbf{a}}\right|_{\Gamma_{n}^{r}}$ is norm-continuous for each $n \in \mathbb{N}$.

Proof: Consider $a \in A_{+}$to be an element satisfying $r^{\mathbf{a}} \leq z_{\mathbf{a}} a \leq 1$. Set $r_{j}:=r^{\mathbf{a}}\left(\omega_{j}\right)$ and $a_{j}:=a\left(\omega_{j}\right)$ for $j \in J \cup\{\infty\}$.

(a) By (O3), one has $r_{j}-r_{j} r_{\infty} \stackrel{\text { SOT }}{\longrightarrow} 0$. As $\left\{\left\|r_{j}-r_{j} r_{\infty}\right\|\right\}_{j \in J}$ is bounded, the SOT-convergence implies $\left\|\left(r_{j}-r_{j} r_{\infty}\right) y\right\| \rightarrow 0$ for any $y \in \mathcal{K}$. Since $r_{j} a_{j}=r_{j}(j \in J \cup\{\infty\})$ and $\left\|a_{j}-a_{\infty}\right\| \rightarrow 0$, we see that

$$
\left\|r_{j}-r_{j} r_{\infty} a_{j}\right\| \leq\left\|\left(r_{j}-r_{j} r_{\infty}\right)\left(a_{j}-a_{\infty}\right)\right\|+\left\|\left(r_{j}-r_{j} r_{\infty}\right) a_{\infty}\right\| \rightarrow 0,
$$

and $\left\|r_{\infty} a_{j}-r_{\infty}\right\|=\left\|r_{\infty} a_{j}-r_{\infty} a_{\infty}\right\| \rightarrow 0$. Thus, the conclusion follows.

(b) Suppose that the statement is not true. By passing to a subnet if necessary, we may assume that $\operatorname{rank} r_{j}>n_{0}:=\operatorname{rank} r_{\infty}$ for all $j \in J$. Let $\left\{\xi_{j}^{(1)}, \ldots, \xi_{j}^{\left(n_{0}+1\right)}\right\}$ be an orthonormal 
subset of $r_{j}\left(\ell^{2}\right)(j \in J)$. Define $\zeta_{j}^{(1)}:=r_{\infty}\left(\xi_{j}^{(1)}\right)$ and put $\zeta_{j}^{(l)}:=r_{\infty}\left(\xi_{j}^{(l)}\right)-\Delta_{j}^{(l)}\left(r_{\infty}\left(\xi_{j}^{(l)}\right)\right)$ for $l=2, \ldots, n_{0}+1$, where $\Delta_{j}^{(l)}$ is the orthogonal projection onto the subspace spanned by $\left\{r_{\infty}\left(\xi_{j}^{(1)}\right), \ldots, r_{\infty}\left(\xi_{j}^{(l-1)}\right)\right\}$. Since $\left\|r_{j}-r_{\infty} r_{j}\right\| \rightarrow 0$ (because of part (a)), for each $\epsilon>0$, one can find $j_{\epsilon} \in J$ such that if $j \geq j_{\epsilon}$ and $\eta \in r_{j}\left(\ell^{2}\right)$, one has $\left\|\eta-r_{\infty} \eta\right\|<\epsilon\|\eta\|$. Thus,

$$
\left\|\zeta_{j}^{(1)}-\xi_{j}^{(1)}\right\|<\epsilon \quad \text { and } \quad\left\|\zeta_{j}^{(1)}\right\| \geq 1-\epsilon \quad\left(j \geq j_{\epsilon}\right) .
$$

Hence, $\left\|\zeta_{j}^{(1)}\right\|>0$ when $\epsilon$ is small enough. Moreover, as

$$
\zeta_{j}^{(2)}=r_{\infty}\left(\xi_{j}^{(2)}\right)-\frac{\left\langle\zeta_{j}^{(1)}, r_{\infty}\left(\xi_{j}^{(2)}\right)\right\rangle}{\left\|\zeta_{j}^{(1)}\right\|^{2}} \zeta_{j}^{(1)}=r_{\infty}\left(\xi_{j}^{(2)}\right)-\frac{\left\langle r_{\infty}\left(\xi_{j}^{(1)}\right)-\xi_{j}^{(1)}, \xi_{j}^{(2)}\right\rangle}{\left\|\zeta_{j}^{(1)}\right\|^{2}} \zeta_{j}^{(1)}
$$

(because $\left\langle\xi_{j}^{(1)}, \xi_{j}^{(2)}\right\rangle=0$ ), one has $\left\|\zeta_{j}^{(2)}-\xi_{j}^{(2)}\right\|<\epsilon+\epsilon /(1-\epsilon)$ and $\left\|\zeta_{j}^{(2)}\right\| \geq 1-\epsilon-\epsilon /(1-\epsilon)$ if $j \geq j_{\epsilon}$. Again, $\left\|\zeta_{j}^{(2)}\right\|>0$ when $\epsilon$ is small enough and the linear span of $\left\{r_{\infty}\left(\xi_{j}^{(1)}\right), r_{\infty}\left(\xi_{j}^{(2)}\right)\right\}$ equals that of $\left\{\zeta_{j}^{(1)}, \zeta_{j}^{(2)}\right\}$. Similarly, $\left\|\zeta_{j}^{(3)}-\xi_{j}^{(3)}\right\|<\epsilon+\frac{\epsilon}{1-\epsilon}+\frac{\epsilon+\epsilon /(1-\epsilon)}{1-\epsilon-\epsilon /(1-\epsilon)}$ and $\left\|\zeta_{j}^{(3)}\right\| \geq 1-\epsilon-$ $\frac{\epsilon}{1-\epsilon}-\frac{\epsilon+\epsilon /(1-\epsilon)}{1-\epsilon-\epsilon /(1-\epsilon)}$ if $j \geq j_{\epsilon}$. Inductively, when $\epsilon$ is small enough, the subset $\left\{\zeta_{j}^{(1)}, \ldots, \zeta_{j}^{\left(n_{0}+1\right)}\right\}$ (for $j \geq j_{\epsilon}$ ) consists of non-zero orthogonal elements in $r_{\infty}\left(\ell^{2}\right)$ which is a contradiction.

(c) Since $a(\omega)$ is compact $(\omega \in \Omega)$, we have $\Gamma_{\infty}^{r}=\emptyset$. Moreover, part (b) tells us that $\bigcup_{k \geq n} \Gamma_{k}^{r}$ is a closed subset of $\Omega$ for $n \in \mathbb{N}$. Finally, as $a(\omega) \leq 1 / 2$ whenever $\omega$ is outside some compact set $K$, we see that $\bigcup_{n \in \mathbb{N}} \Gamma_{n}^{r} \subseteq K$.

(d) Suppose on the contrary that there is a strictly increasing sequence $\left\{n_{k}\right\}_{k \in \mathbb{N}}$ in $\mathbb{N}$ such that each $\Gamma_{n_{k}}^{r}$ contains an element $\gamma_{k}$. By part (c), one can find a subnet of $\left\{\gamma_{k}\right\}_{k \in \mathbb{N}}$ that converges to some $\gamma_{0}$ in $K$ with $\operatorname{rank} r^{\mathbf{a}}\left(\gamma_{0}\right)<\infty$. But this will then contradict part (b).

(e) Suppose that $\omega_{\infty}, \omega_{j} \in \Gamma_{n}^{r}$ for all $j \in J$. Let $\left\{\xi_{j}^{(1)}, \ldots, \xi_{j}^{(n)}\right\}$ be an orthonormal basis of $r_{j}\left(\ell^{2}\right)$, and $\left\{\zeta_{j}^{(1)}, \ldots, \zeta_{j}^{(n)}\right\} \subseteq r_{\infty}\left(\ell^{2}\right)$ be the elements constructed from $\left\{\xi_{j}^{(1)}, \ldots, \xi_{j}^{(n)}\right\}$ as in the proof of part (b). When $j$ is large enough, the argument of part (b) implies that $\eta_{j}^{(l)}:=\zeta_{j}^{(l)} /\left\|\zeta_{j}^{(l)}\right\|(l=1, \ldots, n)$ is well-defined and so $\left\{\eta_{j}^{(1)}, \ldots, \eta_{j}^{(n)}\right\}$ is an orthonormal basis of $r_{\infty}\left(\ell^{2}\right)$. Moreover, the argument of part (b) tells us that $\left\|\zeta_{j}^{(l)}-\xi_{j}^{(l)}\right\| \rightarrow 0(l=1, \ldots, n)$. Consequently, the projection $r_{j}=\sum_{l=1}^{n} \theta_{\xi_{j}^{(l)}, \xi_{j}^{(l)}}$ will norm converge to $r_{\infty}=\sum_{l=1}^{n} \theta_{\eta_{j}^{(l)}, \eta_{j}^{(l)}}$.

Observe that the second countability assumption of $\Omega$ is not actually required in Corollary 4.2 (since the part of Proposition 4.1 that is used in the proof do not require the second countability). Moreover, by the argument of part (b), one may realise part (a) as saying that the net of subspaces $\left\{r^{\mathbf{a}}\left(\omega_{j}\right)\left(\ell^{2}\right)\right\}_{j \in I}$ is "asymptotically contained" in $r^{\mathbf{a}}\left(\omega_{\infty}\right)\left(\ell^{2}\right)$. In the simple situation as in Example 4.4(d) below, the compactness of $r$ is actually equivalent to the condition in part (a) as well as part (c) above. 
Corollary 4.3. Let $A=C_{0}(\Omega) \otimes \mathcal{K}$, and let $p \in \mathrm{OP}(A)$. Set

$$
\Omega_{0}:=\bigcup_{n \in \mathbb{N}_{0}} \operatorname{Int} \Gamma_{n}^{p}
$$

(a) If $n \in \mathbb{N}$ and $\left\{\omega_{j}\right\}_{j \in J}$ is a net in $\Omega$ converging to $\omega_{\infty} \in \Gamma_{n}^{p}$, then for large enough $j \in J$, one can find a rank $n$ subprojection $Q_{j}$ of $p^{\mathbf{a}}\left(\omega_{j}\right)$ such that $\left\|Q_{j}-p^{\mathbf{a}}\left(\omega_{\infty}\right)\right\| \rightarrow 0$.

(b) For a fixed $\omega_{\infty} \in \Omega_{0}$, the map $\omega \mapsto p^{\mathbf{a}}(\omega) p^{\mathbf{a}}\left(\omega_{\infty}\right)$ is norm-continuous at $\omega_{\infty}$, and the restriction $\left.p^{\mathbf{a}}\right|_{\Gamma_{n}^{p}}$ is a norm-continuous function.

(c) $\left.\bar{p}^{\mathbf{a}}\right|_{\Omega_{0}}=\left.p^{\mathbf{a}}\right|_{\Omega_{0}}$ and $\chi_{\Omega_{0}} \cdot p^{\mathbf{a}}=p_{0}^{\mathbf{a}}$ for a unique $p_{0} \in \mathrm{OP}(A)$.

(d) If $\Gamma_{\infty}^{p} \backslash$ Int $\Gamma_{\infty}^{p}$ is a nowhere dense subset of $\Omega$, then $\Omega_{0} \cup \operatorname{Int} \Gamma_{\infty}^{p}$ is dense in $\Omega$.

(e) If $\Gamma_{\infty}^{p}=\emptyset$, then $\bar{p}=\overline{p_{0}}$, where $p_{0} \in \mathrm{OP}(A)$ is as in part (c).

(f) If $p_{0} \in \mathrm{OP}(A)$ is as in part (c), then $\bar{p}$ is compact if and only if $\Gamma_{\infty}^{p}=\emptyset$ and $\overline{p_{0}}$ is compact.

Proof: (a) Consider $\left\{\xi^{(1)}, \ldots, \xi^{(n)}\right\}$ to be an orthonormal basis of $p^{\mathbf{a}}\left(\omega_{\infty}\right)\left(\ell^{2}\right)$. For each $j \in J$, we set $p_{j}:=p^{\mathbf{a}}\left(\omega_{j}\right), \xi_{j}^{(1)}:=p_{j}\left(\xi^{(1)}\right)$ and $\xi_{j}^{(k)}:=p_{j}\left(\xi^{(k)}\right)-\Delta_{j}^{(k)}\left(p_{j}\left(\xi^{(k)}\right)\right)$, where $k \in\{2, \ldots, n\}$ and $\Delta_{j}^{(k)}$ is the projection onto the subspace spanned by $\left\{p_{j}\left(\xi^{(1)}\right), \ldots, p_{j}\left(\xi^{(k-1)}\right)\right\}$. Since $p_{j} p^{\mathbf{a}}\left(\omega_{\infty}\right) \stackrel{S O T}{\longrightarrow} p^{\mathbf{a}}\left(\omega_{\infty}\right)$ (by Proposition 4.1 ), for any $\epsilon>0$, there is $j_{\epsilon} \in J$ such that

$$
\left\|p_{j}\left(\xi^{(k)}\right)-\xi^{(k)}\right\|<\epsilon \quad\left(k=1, \ldots, n ; j \geq j_{\epsilon}\right) .
$$

Now, using a similar argument as that for Corollary $4.2(\mathrm{~b})$, we know that $\left\|\xi_{j}^{(k)}-\xi^{(k)}\right\| \rightarrow 0$ for $k \in\{1, \ldots, n\}$. Thus, $\eta_{j}^{(k)}:=\frac{\xi_{j}^{(k)}}{\left\|\xi_{j}^{(k)}\right\|}(k=1, \ldots, n)$ is well-defined for large enough $j \in J$ and they form an orthonormal subset of $p_{j}\left(\ell^{2}\right)$ such that $\eta_{j}^{(k)} \stackrel{\|\cdot\|}{\longrightarrow} \xi^{(k)}(k=1, \ldots, n)$. Consequently, $Q_{j}:=\sum_{k=1}^{n} \theta_{\eta_{j}^{(k)}, \eta_{j}^{(k)}}$ will norm converge to $p^{\mathbf{a}}\left(\omega_{\infty}\right)=\sum_{k=1}^{n} \theta_{\xi^{(k)}, \xi^{(k)}}$.

(b) The first conclusion follows from Proposition 4.1 as well as the fact that $\operatorname{rank} p^{\mathbf{a}}\left(\omega_{\infty}\right)<\infty$. The second conclusion follows directly from part (a).

(c) By part (b), we know that $\left.p^{\mathbf{a}}\right|_{\Omega_{0}}$ is norm-continuous. It is clear that $h:=\chi_{\Omega_{0}} \cdot p^{\mathbf{a}}$ satisfies the condition in Proposition 4.1, and $h=z_{\mathbf{a}} p_{0}$ for a unique $p_{0} \in \mathrm{OP}(A)$. On the other hand, if we set

$$
f(\omega):= \begin{cases}p^{\mathbf{a}}(\omega) & \text { if } \omega \in \Omega_{0}, \\ \operatorname{id}_{\ell^{2}} & \text { otherwise, }\end{cases}
$$

then part (b) and (O3) tell us that $f$ is a $q$-closed projection. As $p^{\mathbf{a}} \leq f$, we know that $h \leq p^{\mathbf{a}} \leq \bar{p}^{\mathbf{a}} \leq f$, which gives the assertion that $\left.\bar{p}^{\mathbf{a}}\right|_{\Omega_{0}}=\left.p^{\mathbf{a}}\right|_{\Omega_{0}}$.

(d) Assume on the contrary that there is a non-empty element $U$ in $\mathcal{O}(\Omega)$ disjoint from $\Omega_{0} \cup \operatorname{Int} \Gamma_{\infty}^{p}$. Let us first construct the following decreasing sequence of sets:

$$
\Lambda_{0}:=\Omega \quad \text { and } \quad \Lambda_{n}:=\bigcup_{k \geq n} \Gamma_{k}^{p} \cup \Gamma_{\infty}^{p} \quad(n \in \mathbb{N})
$$


Then $\Lambda_{k} \in \mathcal{O}(\Omega)$ (see $\left.(\mathrm{O} 2)\right)$ and $\Gamma_{k}^{p}=\Lambda_{k} \backslash \Lambda_{k+1}\left(k \in \mathbb{N}_{0}\right)$. Thus, Int $\Gamma_{k}^{p}=\Lambda_{k} \backslash \overline{\Lambda_{k+1}}$ and $\partial \Lambda_{k}=\overline{\Lambda_{k}} \backslash \Lambda_{k}$. We claim that

$$
\operatorname{Int} \Gamma_{\infty}^{p} \cup \Omega_{0}=\operatorname{Int} \Gamma_{\infty}^{p} \cup \bigcup_{k \in \mathbb{N}_{0}} \Lambda_{k} \backslash \overline{\Lambda_{k+1}}=\Omega \backslash\left(\left(\Gamma_{\infty}^{p} \backslash \operatorname{Int} \Gamma_{\infty}^{p}\right) \cup \bigcup_{n \in \mathbb{N}} \partial \Lambda_{n}\right) .
$$

Indeed, the first equality is clear. Let $x$ be an element in the disjoint union $\bigcup_{k \in \mathbb{N}_{0}} \Lambda_{k} \backslash \overline{\Lambda_{k+1}}$. Then $x \in \Lambda_{k}$ but $x \notin \overline{\Lambda_{k+1}}$ for a unique $k \in \mathbb{N}_{0}$. Thus, $x \notin \Gamma_{\infty}^{p} \subseteq \bigcap_{l \in \mathbb{N}_{0}} \overline{\Lambda_{l}}$. Moreover, if there exists $l \in \mathbb{N}$ such that $x \in \partial \Lambda_{l}=\overline{\Lambda_{l}} \backslash \Lambda_{l} \subseteq \overline{\Lambda_{l}}$, then $l \leq k$, which gives a contradiction that $x \notin \Lambda_{k}$ (because $x \notin \Lambda_{l}$ ). On the other hand, let $x \in \operatorname{Int} \Gamma_{\infty}^{p}$. Then $x \in \Lambda_{n}$ and hence $x \notin \partial \Lambda_{n}=\overline{\Lambda_{n}} \backslash \Lambda_{n}$, for all $n \in \mathbb{N}$. These give one of the inclusions. Conversely, suppose that $x \notin\left(\Gamma_{\infty}^{p} \backslash \operatorname{Int} \Gamma_{\infty}^{p}\right) \cup \bigcup_{n \in \mathbb{N}}\left(\overline{\Lambda_{n}} \backslash \Lambda_{n}\right)$. If $x \in \Gamma_{\infty}^{p}$, then $x \in \operatorname{Int} \Gamma_{\infty}^{p}$. If $x \notin \Gamma_{\infty}^{p}$, there is a smallest $k \in \mathbb{N}$ such that $x \notin \Lambda_{k}$, which implies that $x \notin \overline{\Lambda_{k}}$. Thus, $x \in \Lambda_{k-1} \backslash \overline{\Lambda_{k}}$. These establish the other inclusion.

From the claim above, we know that $\overline{\Gamma_{\infty}^{p} \backslash \operatorname{Int} \Gamma_{\infty}^{p}} \cup \bigcup_{n \in \mathbb{N}} \partial \Lambda_{n}$ contains $U$. As $\Gamma_{\infty}^{p} \backslash \operatorname{Int} \Gamma_{\infty}^{p}$ is nowhere dense and each $\partial \Lambda_{n}$ is a closed subset of $\Omega$, we see that there exists $n_{0} \in \mathbb{N}$ with $\partial \Lambda_{n_{0}}$ having non-empty interior (notice that $\Omega$ is a Baire space) which contradicts the fact that $\Lambda_{n_{0}} \in \mathcal{O}(\Omega)$.

(e) Since $p_{0} \leq p$, we have $\overline{p_{0}} \leq \bar{p}$, and part (c) tells us that

$$
\left.p^{\mathbf{a}}\right|_{\Omega_{0}}=\left.{\overline{p_{0}}}^{\mathbf{a}}\right|_{\Omega_{0}}=\left.\bar{p}^{\mathbf{a}}\right|_{\Omega_{0}} .
$$

On the other hand, let $\omega_{\infty} \in \Omega \backslash \Omega_{0}$. By part (d), $\Omega_{0}$ is dense in $\Omega$ and we have a net $\left\{\omega_{j}\right\}_{j \in J}$ in $\Omega_{0}$ that converges to $\omega_{\infty}$. By part (a), we may assume that for any $j \in J$, there is a rank $n$ subprojection $Q_{j}$ of $p^{\mathbf{a}}\left(\omega_{j}\right)$ such that $\left\|Q_{j}-p^{\mathbf{a}}\left(\omega_{\infty}\right)\right\| \rightarrow 0$. On the other hand, (O3) and (4.3) tells us that $p^{\mathbf{a}}\left(\omega_{j}\right) \overline{p_{0}} \mathbf{a}\left(\omega_{\infty}\right)-p^{\mathbf{a}}\left(\omega_{j}\right) \stackrel{\text { SOT }}{\longrightarrow} 0$. Consequently, $\left\{Q_{j} \bar{p}_{0} \mathbf{a}\left(\omega_{\infty}\right)-Q_{j}\right\}_{j \in J}$ will both SOT-converge to 0 and norm-converge to $p^{\mathbf{a}}\left(\omega_{\infty}\right) \overline{p_{0}} \mathbf{a}\left(\omega_{\infty}\right)-p^{\mathbf{a}}\left(\omega_{\infty}\right)$. This implies that $p^{\mathbf{a}}\left(\omega_{\infty}\right) \leq{\overline{p_{0}}}^{\mathbf{a}}\left(\omega_{\infty}\right)$, and one has $p \leq \overline{p_{0}}$. As $\overline{p_{0}}$ is closed, we see that $\bar{p} \leq \overline{p_{0}}$.

(f) This follows directly from part (e) and Corollary 4.2(c).

If $r$ is a closed projection, $\omega_{\infty} \in \Omega$ with $\operatorname{rank}(1-r)^{\mathbf{a}}\left(\omega_{\infty}\right)<\infty$ and $\left\{\omega_{i}\right\}_{i \in I}$ is a net in $\Omega$ converging to $\omega_{\infty}$, the same reasoning for Corollary 4.3(b) also implies that $\| r^{\mathbf{a}}\left(\omega_{j}\right)-$ $r^{\mathbf{a}}\left(\omega_{j}\right) r^{\mathbf{a}}\left(\omega_{\infty}\right) \| \rightarrow 0$. However, one has rank $(1-r)^{\mathbf{a}}\left(\omega_{\infty}\right)=\infty$ when $r$ is compact (by Corollary 4.2(c)), and the conclusion of Corollary 4.2(a) does not follow from Corollary $4.3(\mathrm{~b})$.

Corollary 4.3(e) tells us that if $\operatorname{rank} p^{\mathbf{a}}(\omega)<\infty$ for all $\omega \in \Omega$, then $\bar{p}^{\mathbf{a}}$ coincides with the "q-closure" of $\left.p^{\mathbf{a}}\right|_{\Omega_{0}}$. 
Example 4.4. Let $\overline{\mathbb{N}}$ be the one point compactification of $\mathbb{N}$. Set $B:=C(\overline{\mathbb{N}}) \otimes \mathcal{K}$ as well as $D:=C(\overline{\mathbb{N}}) \otimes M_{2}$. It is well-known that $B^{* *} \cong \ell^{\infty}(\overline{\mathbb{N}} ; \mathcal{B})$ and $D^{* *} \cong \ell^{\infty}\left(\overline{\mathbb{N}} ; M_{2}\right)$.

(a) As $D$ is canonically a hereditary $C^{*}$-subalgebra of $B$, Proposition 4.1 and (H1) implies that a map $p: \overline{\mathbb{N}} \rightarrow \operatorname{Proj}\left(M_{2}\right)$ belongs to $\mathrm{OP}(D)$ if and only if one of the following is satisfied:

i). $p(\infty)=p(k)=\mathrm{id}_{\mathbb{C}^{2}}$ for large enough $k$;

ii). $\operatorname{rank} p(\infty)=1, \operatorname{rank} p(k) \geq 1$ eventually and if $\{p(k): \operatorname{rank} p(k)=1\}$ is infinite, then it is a sequence that converges to $p(\infty)$;

iii). $p(\infty)=0$.

By direct verifications, for any $p, q \in \mathrm{OP}(D)$, one has $p \sim_{\mathrm{Mv}} q$ if and only if $p \sim_{\mathrm{PZ}} q$.

(b) For any $p \in \mathrm{OP}(D)$, the description (O3) for closed projections tells us that one of the following holds:

I). If $\left\{k \in \mathbb{N}: p(k)=\mathrm{id}_{\mathbb{C}^{2}}\right\}$ is infinite, then $\bar{p}(\infty)=\mathrm{id}_{\mathbb{C}^{2}}$.

II). If $p(k) \rightarrow p(\infty)$, then $\bar{p}(\infty)=p(\infty)$.

III). If $p(\infty)=0, \operatorname{rank} p(k) \leq 1$ eventually and $\{p(k): \operatorname{rank} p(k)=1\}$ forms an infinite sequence that converges to some $q$, then $\bar{p}(\infty)=q$.

IV). If $p(\infty)=0, \operatorname{rank} p(k) \leq 1$ eventually and $\{p(k): \operatorname{rank} p(k)=1\}$ forms an infinite non-Cauchy sequence, then $\bar{p}(\infty)=\mathrm{id}_{\mathbb{C}^{2}}$.

As I) - IV) exhaust all the possibilities of closures of open projections of $D$, we know, from (O3), that a closed projection $r$ of $D$ is not the closure of any open projection if and only if $r(\infty)=\mathrm{id}_{\mathbb{C}^{2}}$, rank $r(k) \leq 1$ eventually, and either $r(k)=0$ eventually or $\{r(k): \operatorname{rank} r(k)=1\}$ forms an infinite convergent sequence.

(c) Set $f_{n}^{(k)}:=\left\{\begin{array}{ll}0 & \text { if } k \leq n(n-1) / 2 \text { or } k>n(n+1) / 2 \\ 1 & \text { if } n(n-1) / 2<k \leq n(n+1) / 2\end{array}\right.$. Let $r_{n}$ be the diagonal matrix with diagonal $\left(f_{n}^{(1)}, f_{n}^{(2)}, \ldots\right)$, considered as a projection in $\mathcal{B}$. Then $\operatorname{rank} r_{n}=n$ and $r_{n} \stackrel{S O T}{\longrightarrow} 0$. Thus, $r:=\left(\left(r_{n}\right)_{n \in \mathbb{N}}, 0\right)$ is a closed projection of $B$ by (O3). Note, however, that as $\Gamma_{k}^{r}=\{k\}$ $(k \in \mathbb{N})$ and $\Gamma_{\infty}^{r}=\emptyset$, the set $\bigcup_{k \geq 1} \Gamma_{k}^{r} \cup \Gamma_{\infty}^{r}$ is not closed in $\overline{\mathbb{N}}$ (c.f. the contrast with (O2) and Corollary 4.2(c)).

(d) If $r \in \operatorname{Proj}\left(B^{* *}\right)$, then $r$ is compact if and only if

$$
\operatorname{rank} r(k)<\infty \quad(k \in \overline{\mathbb{N}}) \quad \text { and } \quad\|r(k)-r(k) r(\infty)\| \rightarrow 0 .
$$

In fact, we only need to establish the sufficiency in view of Corollary 4.2(a). Suppose that $r$ satisfies the two conditions as displayed. By (O3), we know that $r$ is a closed projection. Set $a(k):=r_{k}+\left(1-r_{k}\right) r_{\infty}\left(1-r_{k}\right) \in \mathcal{K}_{+}$, where $r_{k}:=r(k)(k \in \overline{\mathbb{N}})$. Since

$$
r_{k}+\left(1-r_{k}\right) r_{\infty}\left(1-r_{k}\right)=r_{\infty}+r_{k}-r_{k} r_{\infty}+\left(r_{k} r_{\infty}-r_{k}\right) r_{k}+r_{k}-r_{\infty} r_{k},
$$

we see that $\|a(k)-a(\infty)\| \leq 3\left\|r_{k}-r_{k} r_{\infty}\right\| \rightarrow 0$. Thus, $a \in B_{+}$and $r a=r$ as required. 


\section{Comparison of equivalence Relations on $\mathrm{OP}\left(C_{0}(\Omega) \otimes \mathcal{K}\right)$}

In this section, we compare some equivalence relations on $\mathrm{OP}\left(C_{0}(\Omega ; \mathcal{K})\right)$. Before we present our first lemma in this section, let us set some notations. Let $n \in \overline{\mathbb{N}}$ and $\mathbb{C}^{n}$ be the $n$ dimensional Hilbert space (we identify $\mathbb{C}^{\infty}$ with $\ell^{2}$ in the canonical way). Suppose that $\mathcal{J}_{n}$ is the set of isometries in $\mathcal{B}\left(\mathbb{C}^{n} ; \ell^{2}\right), \mathcal{P}_{n}$ is the set of rank $n$ projections in $\mathcal{B}$, and $\mathcal{U}_{n}$ is the set of unitaries in $\mathcal{B}\left(\mathbb{C}^{n}\right)$. In the following, we will equip $\mathcal{J}_{n}, \mathcal{P}_{n}$ and $\mathcal{U}_{n}$ with the WOT, which coincides with the corresponding SOT.

Define a map $\mathcal{F}_{n}: \mathcal{J}_{n} \rightarrow \mathcal{P}_{n}$ by

$$
\mathcal{F}_{n}(T):=T T^{*} \quad\left(T \in \mathcal{J}_{n}\right) .
$$

It is not hard to check that $\mathcal{F}_{n}$ is continuous when $n<\infty$. We will see in the following lemma that $\mathcal{F}_{n}$ is a Borel map (for $n \in \overline{\mathbb{N}}$ ) and it has a Borel right inverse (i.e. a Borel map $\psi_{n}: \mathcal{P}_{n} \rightarrow \mathcal{J}_{n}$ satisfying $\left.\mathcal{F}_{n} \circ \psi_{n}=\mathrm{id}_{\mathcal{P}_{n}}\right)$. This lemma could be a known fact, but we do not find it explicitly stated in the literature. We give a proof here for completeness.

Lemma 5.1. Let $n \in \overline{\mathbb{N}}$.

(a) We equip $\mathcal{B}$ with the WOT and consider $\mathcal{G}_{n}: \mathcal{J}_{n} \times \mathcal{J}_{n} \rightarrow \mathcal{B}$ to be the map given by $(S, T) \mapsto S T^{*}$. If $n<\infty$, then $\mathcal{G}_{n}$ is continuous. Moreover, $\mathcal{G}_{\infty}$ is Borel.

(b) There exists a Borel right inverse $\psi_{n}: \mathcal{P}_{n} \rightarrow \mathcal{J}_{n}$ for $\mathcal{F}_{n}$.

Proof: Let $\left\{\varsigma^{(l)}\right\}_{l \in \mathbb{N}}$ be an orthonormal basis for $\ell^{2}$. For a fixed $k \in \mathbb{N}$, we consider the projection $e_{k}$ from $\ell^{2}$ onto the linear span of $\left\{\varsigma^{(l)}, \ldots, \varsigma^{(k)}\right\}$.

(a) The first claim is clear. For the second claim, we note first of all, that $(S, T) \mapsto S T^{*} e_{k}$ is a continuous map from $\mathcal{J}_{\infty} \times \mathcal{J}_{\infty}$ to $\mathcal{B}$ (recall that WOT coincides with SOT on $\mathcal{J}_{\infty}$ ). Thus, for any $\xi, \eta \in \ell^{2}$, the map $(S, T) \mapsto\left\langle T^{*} e_{k} \xi, S^{*} e_{k} \eta\right\rangle$ is continuous, which implies that $(S, T) \mapsto\left\langle S T^{*} \xi, \eta\right\rangle$ is Borel, because $\left\langle T^{*} \xi, S^{*} \eta\right\rangle=\lim _{k \rightarrow \infty}\left\langle T^{*} e_{k} \xi, S^{*} e_{k} \eta\right\rangle$.

(b) Consider the continuous action $\gamma$ of $\mathcal{U}_{n}$ on $\mathcal{J}_{n}$ given by $\gamma_{U}(T):=T U^{*}\left(U \in \mathcal{U}_{n} ; T \in \mathcal{J}_{n}\right)$. Clearly, $\left\{\mathcal{F}_{n}^{-1}(P): P \in \mathcal{P}_{n}\right\}$ coincides with the set of $\gamma$-orbits. These give a bijection $\hat{\mathcal{F}}_{n}: \mathcal{J}_{n} / \gamma \rightarrow \mathcal{P}_{n}$.

Case 1: $n<\infty$.

The proof of Corollary 4.3(a) tells us that if $\left\{P_{j}\right\}_{j \in \mathbb{N}}$ is a sequence in $\mathcal{P}_{n}$ that converges to $P \in \mathcal{P}_{n}$ with respects to WOT and $\left\{\xi^{(1)}, \ldots, \xi^{(n)}\right\}$ is an orthonormal basis of $P\left(\ell^{2}\right)$, then for large enough $j$, one can find an orthonormal basis $\left\{\xi_{j}^{(1)}, \ldots, \xi_{j}^{(n)}\right\}$ of $P_{j}\left(\ell^{2}\right)$ such that $\left\|\xi_{j}^{(k)}-\xi^{(k)}\right\| \rightarrow 0$ for all $k=1, \ldots, n$. This shows that $\hat{\mathcal{F}}_{n}^{-1}: \mathcal{P}_{n} \rightarrow \mathcal{J}_{n} / \gamma$ is continuous. Note that $\mathcal{U}_{n}$ is a separable compact metrizable group, $\mathcal{J}_{n}$ is a Polish space under the norm topology (which coincides with WOT as $n<\infty$ ) and the stabilizer subgroup of $\mathcal{U}_{n}$ for each 
$T \in \mathcal{J}_{n}$ is trivial. It then follows from [8, Theorem 2.9] that there is a Borel cross section $\varphi_{n}: \mathcal{J}_{n} / \gamma \rightarrow \mathcal{J}_{n}$ and we may take $\psi_{n}:=\varphi_{n} \circ \hat{\mathcal{F}}_{n}^{-1}$.

Case 2: $n=\infty$.

As in the finite dimensional case, we first show that $\hat{\mathcal{F}}_{\infty}^{-1}$ is continuous. Suppose that $\left\{P_{j}\right\}_{j \in \mathbb{N}}$ is a sequence in $\mathcal{P}_{\infty}$ that SOT-converges to $P \in \mathcal{P}_{\infty}$. Consider $\left\{\xi^{(k)}\right\}_{k \in \mathbb{N}}$ to be an orthonormal basis for $P\left(\ell^{2}\right)$. As in the proof of Corollary 4.3(a), for each $j \in \mathbb{N}$, we set $\xi_{j}^{(1)}:=$ $P_{j}\left(\xi^{(1)}\right)$ as well as $\xi_{j}^{(k)}:=P_{j}\left(\xi^{(k)}\right)-\Delta_{j}^{(k)}\left(P_{j}\left(\xi^{(k)}\right)\right)$, where $k \geq 2$ and $\Delta_{j}^{(k)}$ is the projection onto the subspace spanned by $\left\{P_{j}\left(\xi^{(1)}\right), \ldots, P_{j}\left(\xi^{(k-1)}\right)\right\}$. By the SOT-convergence, for each $m \in \mathbb{N}$, one can find a positive integer $j_{m}>j_{m-1}\left(\right.$ with $j_{0}:=1$ ) such that $\left\{\xi_{j}^{(1)}, \ldots, \xi_{j}^{(m)}\right\}$ is a set of non-zero orthogonal vectors whenever $j \geq j_{m}$.

Suppose that $i \in \mathbb{N}$. Then $j_{m_{i}} \leq i<j_{m_{i}+1}$ for some $m_{i} \in \mathbb{N}_{0}$, and we consider $\left\{\eta_{i}^{(l)}\right\}_{l \in \mathbb{N}}$ to be an orthonormal basis for $P_{i}\left(\ell^{2}\right)$ such that $\eta_{i}^{(l)}=\frac{\xi_{i}^{(l)}}{\left\|\xi_{i}^{(l)}\right\|}$ for $l=1, \ldots, m_{i}$. The SOTconvergence implies that $\left\|\eta_{i}^{(l)}-\xi^{(l)}\right\| \rightarrow 0$ for every $l \in \mathbb{N}$. We denote by $Q_{i}$ the projection onto the subspace spanned by $\left\{\eta_{i}^{(1)}, \ldots, \eta_{i}^{\left(m_{i}\right)}\right\}$ and define $R_{i}, S_{i} \in \mathcal{B}$ by

$$
R_{i}\left(\varsigma^{(l)}\right):=\left\{\begin{array}{ll}
\eta_{i}^{(l)} & \text { if } l \leq m_{i} \\
0 & \text { if } l>m_{i}
\end{array} \quad \text { and } \quad S_{i}\left(\varsigma^{(l)}\right):= \begin{cases}0 & \text { if } l \leq m_{i} \\
\eta_{i}^{(l)} & \text { if } l>m_{i}\end{cases}\right.
$$

Then $R_{i}+S_{i} \in \mathcal{J}_{\infty}, R_{i} R_{i}^{*}=Q_{i}$ and $\mathcal{F}_{\infty}\left(R_{i}+S_{i}\right)=P_{i}$. It is easy to see that $Q_{i} \stackrel{\text { SOT }}{\longrightarrow} P$ and $\left\{R_{i}\right\}_{i \in \mathbb{N}}$ will WOT-converge to the isometry $R$ defined by $R\left(\varsigma^{(l)}\right):=\xi^{(l)}(l \in \mathbb{N})$. On the other hand, since $S_{i} S_{i}^{*}=P_{i}-Q_{i} \stackrel{\text { WOT }}{\longrightarrow} 0$, we have $S_{i}^{*} \stackrel{\text { SOT }}{\longrightarrow} 0$ and so $S_{i} \stackrel{\text { WOT }}{\longrightarrow} 0$. Now, $\left\{R_{i}+S_{i}\right\}_{i \in \mathbb{N}}$ is a sequence in $\mathcal{J}_{\infty}$ that WOT-converges to $R$ with $\mathcal{F}_{\infty}(R)=P$. Consequently, $\hat{\mathcal{F}}_{\infty}^{-1}$ is continuous as required.

Secondly, it is well-known that the closed unit ball, $\mathcal{B}_{1}$, of $\mathcal{B}$ is a Polish space under SOT. Thus, $\mathcal{P}_{\infty} \subseteq \mathcal{B}_{1}$ is second countable (recall again that WOT coincides with SOT on $\mathcal{P}_{\infty}$ ) and $\mathcal{J}_{\infty}$ (being SOT-closed in $\mathcal{B}_{1}$ ) is a Polish space. As $\hat{\mathcal{F}}_{\infty}$ is a bijective Borel map (since $\mathcal{F}_{\infty}$ is a Borel map by part (a)), we know that $\mathcal{J}_{\infty} / \gamma$ is countably separated under the quotient Borel structure. Furthermore, by [7, Lemme 4], $\mathcal{U}_{\infty}$ is a Polish group under SOT. One may then apply the main theorem of [6] to obtain a Borel transversal $X \subseteq \mathcal{J}_{\infty}$ for $\gamma$. Now, the argument in [13, Theorem 5.2] (see also $(12) \Rightarrow(13)$ of $[8$, Theorem 2.9]) tells us that there is a Borel cross section $\varphi_{\infty}$ from the quotient Borel space $\mathcal{J}_{\infty} / \gamma$ to $\mathcal{J}_{\infty}$. On the other hand, the argument in [13, Theorem 5.1] (see also $(7) \Rightarrow(6)$ of [8, Theorem 2.9]) tells us that the quotient topology of $\mathcal{J}_{\infty} / \gamma$ generates the quotient Borel structure. Therefore, $\psi_{\infty}:=\varphi_{\infty} \circ \hat{\mathcal{F}}_{\infty}^{-1}$ is a Borel map from $\mathcal{P}_{\infty}$ to $\mathcal{J}_{\infty}$ satisfying the requirement. 
Lemma 5.2. Let $\mathcal{M}(\Omega ; \mathcal{B})$ be the set of all bounded Borel maps from $\Omega$ to $\mathcal{B}$, when $\mathcal{B}$ is equipped with WOT. If $p, q \in \mathrm{OP}\left(C_{0}(\Omega) \otimes \mathcal{K}\right)$ satisfying $\Gamma_{n}^{p}=\Gamma_{n}^{q}(n \in \mathbb{N})$, there is $u \in \mathcal{M}(\Omega ; \mathcal{B})$ with

$$
u u^{*}=p^{\mathbf{a}} \text { and } u^{*} u=q^{\mathbf{a}}
$$

Proof: By Inequality (4.1), we know that for any $\xi \in \ell^{2}$, the function $\omega \mapsto\left\langle p^{\mathbf{a}} \xi, \xi\right\rangle$ is lower semi-continuous and hence Borel. Thus, the polarization identity tells us that $\omega \mapsto\left\langle p^{\mathbf{a}} \xi, \eta\right\rangle$ is a Borel function for any $\xi, \eta \in \ell^{2}$. Consequently, $p^{\mathbf{a}}, q^{\mathbf{a}} \in \mathcal{M}(\Omega ; \mathcal{B})$.

Notice also that $p^{\mathbf{a}}\left(\Gamma_{n}^{p}\right), q^{\mathbf{a}}\left(\Gamma_{n}^{q}\right) \subseteq \mathcal{P}_{n}(n \in \overline{\mathbb{N}})$. Let $\psi_{n}: \mathcal{P}_{n} \rightarrow \mathcal{J}_{n}$ be the Borel right inverse of $\mathcal{F}_{n}$ as given by Lemma $5.1(\mathrm{~b})$. Define

$$
u_{n}(\omega):=\psi_{n}\left(p^{\mathbf{a}}(\omega)\right) \psi_{n}\left(q^{\mathbf{a}}(\omega)\right)^{*} \quad\left(\omega \in \Gamma_{n}^{p}\right) .
$$

Lemma 5.1(a) tells us that $u_{n}$ is a bounded Borel map from $\Gamma_{n}^{p}$ to $\mathcal{B}$ such that $u_{n} u_{n}^{*}=\left.p^{\mathbf{a}}\right|_{\Gamma_{n}^{p}}$ and $u_{n}^{*} u_{n}=\left.q^{\mathbf{a}}\right|_{\Gamma_{n}^{p}}$. Now, we may define $u \in \mathcal{M}(\Omega ; \mathcal{B})$ by setting $u(\omega):=u_{n}(\omega)$ whenever $\omega \in \Gamma_{n}^{p}$, and $u(\omega)=0$ whenever $\omega \in \Gamma_{0}^{p}$.

We let $\mathfrak{M}$ be a maximal family of mutually singular continuous Radon measures on $\Omega$ of norm 1 . As in $\left[5\right.$, p.434] (see also $[4, \S 5 . \mathrm{G}]$ ), there is a canonical ${ }^{*}$-isomorphism $C_{0}(\Omega)^{* *} \cong$ $\ell^{\infty}(\Omega) \oplus_{\ell^{\infty}} \bigoplus_{\mu \in \mathfrak{M}} L^{\infty}(\Omega, \mu)$ and thus, we can identify

$$
\left(C_{0}(\Omega) \otimes \mathcal{K}\right)^{* *}=C_{0}(\Omega)^{* *} \bar{\otimes} \mathcal{B} \cong \ell^{\infty}(\Omega ; \mathcal{B}) \oplus_{\ell \infty} \bigoplus_{\mu \in \mathfrak{M}} L^{\infty}((\Omega, \mu) ; \mathcal{B})
$$

such that the canonical embedding $\kappa_{C_{0}(\Omega) \otimes \mathcal{K}}$ is the one given by the canonical map from $C_{0}(\Omega) \otimes \mathcal{K}$ to $\ell^{\infty}(\Omega ; \mathcal{B})$ as well as those from $C_{0}(\Omega) \otimes \mathcal{K}$ to $L^{\infty}((\Omega, \mu) ; \mathcal{B})(\mu \in \mathfrak{M})$.

For every $\mu \in \mathfrak{M}$, we consider $\Phi_{\mu}: \mathcal{M}(\Omega ; \mathcal{B}) \rightarrow L^{\infty}((\Omega, \mu) ; \mathcal{B})$ to be the canonical *homomorphism and set

$$
\Phi(w):=w+\sum_{\mu \in \mathfrak{M}} \Phi_{\mu}(w) \quad(w \in \mathcal{M}(\Omega ; \mathcal{B})) .
$$

It is easy to see that

$$
\Phi\left(\kappa_{C_{0}(\Omega) \otimes \mathcal{K}}^{\mathbf{a}}(a)\right)=\kappa_{C_{0}(\Omega) \otimes \mathcal{K}}(a) \quad\left(a \in C_{0}(\Omega) \otimes \mathcal{K}\right) .
$$

Now, we can produce an example showing that $\sim_{\text {sp }}$ is different from $\sim_{\mathrm{PZ}}$ on $\operatorname{Proj}(C(\Omega) \otimes \mathcal{K})$ for a compact Hausdorff space $\Omega$ with $\operatorname{dim} \Omega=2$. Notices that in order to obtain this counter example, we only need the easier case of $n<\infty$ in Lemma 5.1 (b) (since $\Gamma_{\infty}^{p}=\Gamma_{\infty}^{q}=\emptyset$ for the two open projections $p$ and $q$ considered in the proof of Theorem 5.3).

Theorem 5.3. There exists $p, q \in \operatorname{Proj}\left(C\left(\mathbb{C P}^{1}\right) \otimes \mathcal{K}\right)$ such that $p \sim_{\mathrm{sp}} q$ but $p \nsim_{\mathrm{PZ}} q$. 
Proof: Let $A:=C\left(\mathbb{C P}^{1} ; \mathcal{K}\right)$ and $B:=C\left(\mathbb{C P}^{1} ; M_{2}\right)$. By fixing an embedding of $M_{2}$ as a hereditary $C^{*}$-subalgebra of $\mathcal{K}$, one can regard $B$ as a hereditary $C^{*}$-subalgebra of $A$. Moreover, we identify $\mathbb{S}^{3}$ with $\left\{\xi \in \mathbb{C}^{2}:\|\xi\|=1\right\}$ and consider $\Lambda: \mathbb{S}^{3} \rightarrow \mathbb{C P}^{1}$ to be the canonical quotient map. Define $p, q: \mathbb{C P}^{1} \rightarrow M_{2} \subseteq \mathcal{K}$ by setting

$$
p(\Lambda(\xi)):=\theta_{\xi, \xi} \quad \text { and } \quad q(\Lambda(\xi)):=\theta_{\eta_{0}, \eta_{0}} \quad\left(\xi \in \mathbb{S}^{3}\right),
$$

where $\eta_{0} \in \mathbb{S}^{3}$ is a fixed vector. Clearly, $p$ is the canonical continuous map from $\mathbb{C P}^{1}$ to $\operatorname{Proj}\left(M_{2}\right)$. Thus, $p, q \in \operatorname{Proj}(A)$.

Lemma 5.2 gives an element $u \in \mathcal{M}\left(\mathbb{C P}^{1} ; \mathcal{B}\right.$ ) satisfying $u u^{*}=p^{\mathbf{a}}$ and $u^{*} u=q^{\mathbf{a}}$ (as $\operatorname{rank} p(\omega)=1=\operatorname{rank} q(\omega)$ for all $\omega \in \Omega)$. For any $x \in \operatorname{her}(p)=p B p=p A p$ and $\omega \in \mathbb{C P}^{1}$, one has $x(\omega)=\lambda(\omega) p(\omega)$ for some $\lambda(\omega) \in \mathbb{C}$. By taking the trace on $M_{2}$, we know that $\lambda$ is continuous on $\mathbb{C P}^{1}$. Since

$$
\left(u^{*} x u\right)(\omega)=\lambda(\omega) q(\omega) \quad\left(\omega \in \mathbb{C P}^{1}\right),
$$

we conclude that $u^{*} \kappa_{A}^{\mathbf{a}}(x) u \in \kappa_{A}^{\mathbf{a}}(\operatorname{her}(q))$. Similarly, $u \kappa_{A}^{\mathbf{a}}(\operatorname{her}(q)) u^{*} \subseteq \kappa_{A}^{\mathbf{a}}(\operatorname{her}(p))$.

Suppose that $\Phi: \mathcal{M}\left(\mathbb{C P}^{1} ; \mathcal{B}\right) \rightarrow A^{* *}$ is the map given by (5.1) and (5.2). Then (5.3) tells us that $\kappa_{A}(b)=\Phi\left(\kappa_{A}^{\mathbf{a}}(b)\right)(b \in B)$, which gives

$$
\Phi(u)^{*} \kappa_{A}(\operatorname{her}(p)) \Phi(u) \subseteq \kappa_{A}(\operatorname{her}(q)) \quad \text { and } \quad \Phi(u) \kappa_{A}(\operatorname{her}(q)) \Phi(u)^{*} \subseteq \kappa_{A}(\operatorname{her}(p)) .
$$

Consequently, $p \sim_{\text {sp }} q$ as elements in $\mathrm{OP}(A)$.

Suppose on the contrary that $p \sim_{\mathrm{PZ}} q$ as elements in $\mathrm{OP}(A)$. By (F5), there is $v \in A$ with $p=v v^{*}$ and $q=v^{*} v$. As $p, q \in B$, we know that actually $v \in B$. Let $\xi(\omega) \in \mathbb{S}^{3}$ satisfying $v(\omega)=\theta_{\eta_{0}, \xi(\omega)}$ and $p(\omega)=\theta_{\xi(\omega), \xi(\omega)}$. Since $v \in C\left(\mathbb{C P}^{1} ; M_{2}\right)$, we see that $\xi: \mathbb{C P}^{1} \rightarrow \mathbb{S}^{3}$ is a continuous map satisfying $\Lambda \circ \xi=\mathrm{id}_{\mathbb{C P}^{1}}$. However, this is impossible because the second homology group $H_{2}\left(\mathbb{C P}^{1}\right)=\mathbb{Z}$, while $H_{2}\left(\mathbb{S}^{3}\right)=(0)$.

Note that in Theorem 5.3 we also have $p \sim_{\text {sp }} q$ as elements in $\mathrm{OP}(B)$ (because of $(\mathrm{H} 3)$ ). On the other hand, the obstruction for $p \sim_{\mathrm{PZ}} q$ in the above is the fact that there is no continuous right inverse of the map $\Lambda: \mathbb{S}^{3} \rightarrow \mathbb{C P}^{1}$, although there always exists a Borel right inverse due to Lemma 5.1(b). In Corollary 5.7(b) below, we will see that the existence of such a continuous right inverse ties up very closely with the PZ-equivalence.

In the following, we will give some more comparisons among $\sim_{\mathrm{Mv}}, \sim_{\mathrm{Cu}}, \sim_{\mathrm{PZ}}$ and $\sim_{\mathrm{sp}}$. These comparisons are related to the stabilized Cuntz semigroup of $C_{0}(\Omega)$. Let us first recall from $[18, \S 6.1]$ that one may consider the stabilized Cuntz semigroup of a separable $C^{*}$-algebra $B$ as the set

$$
\mathrm{Cu}(B)=\mathrm{OP}(B \otimes \mathcal{K}) / \sim_{\mathrm{Cu}}
$$

equipped with the canonical semigroup structure. 
From now on, we set $A:=C_{0}(\Omega) \otimes \mathcal{K}$.

For any $p \in \mathrm{OP}(A)$, we obtain, from (O2), that the function $\omega \mapsto \operatorname{rank} p^{\mathbf{a}}(\omega)$ is lower semicontinuous. This gives a map $\hat{\Theta}: \operatorname{OP}(A) \rightarrow \operatorname{Lsc}\left(\Omega ; \overline{\mathbb{N}}_{0}\right)$ (the set of lower semicontinuous functions from $\Omega$ to $\overline{\mathbb{N}}_{0}$ ) satisfying

$$
\hat{\Theta}(p)(\omega):=\operatorname{rank} p^{\mathbf{a}}(\omega) \quad(p \in \mathrm{OP}(A) ; \omega \in \Omega) .
$$

If $p, q \in \mathrm{OP}(A)$ with $p \sim_{\mathrm{Cu}} q$, then $p \sim_{\mathrm{Mv}} q$ which clearly implies $\hat{\Theta}(p)=\hat{\Theta}(q)$. Thus, we obtain a map $\Theta: \operatorname{Cu}\left(C_{0}(\Omega)\right) \rightarrow \operatorname{Lsc}\left(\Omega, \overline{\mathbb{N}}_{0}\right)$. The following proposition could be known to experts. It tells us that $\hat{\Theta}(p)=\hat{\Theta}(q)$ is actually equivalent to $p \sim_{\mathrm{Mv}} q$.

Proposition 5.4. Suppose that $A=C_{0}(\Omega) \otimes \mathcal{K}$. If $p, q \in \mathrm{OP}(A)$, then $p \sim_{\mathrm{Mv}} q$ if and only if there exists $v \in \ell^{\infty}(\Omega ; \mathcal{B})$ such that $v v^{*}=p^{\mathbf{a}}$ and $v^{*} v=q^{\mathbf{a}}$ (or equivalently, $\operatorname{rank} p^{\mathbf{a}}(\omega)=$ $\operatorname{rank} q^{\mathbf{a}}(\omega)$ for any $\left.\omega \in \Omega\right)$.

Proof: It suffices to verify that $p \sim_{\mathrm{Mv}} q$ if such a $v$ can be found. For any $n \in \overline{\mathbb{N}}$, the existence of $v$ implies that $\Gamma_{n}^{p}=\Gamma_{n}^{q}$. By Lemma 5.2 , one can find $u \in \mathcal{M}(\Omega ; \mathcal{B})$ satisfying

$$
u u^{*}=p^{\mathbf{a}} \text { and } u^{*} u=q^{\mathbf{a}} .
$$

Let $\Phi: \mathcal{M}(\Omega, \mathcal{B}) \rightarrow A^{* *}$ be the map given by (5.1) and (5.2). As $A$ is separable, there exists $b \in A_{+}$with $\left\{\kappa_{A}(b)^{1 / n}\right\}_{n \in \mathbb{N}}$ being $\sigma^{*}$-converging to $p$. For any $f \in \mathcal{K}_{+}^{*}$, one has $f\left(b^{1 / n}(\omega)\right) \uparrow f\left(p^{\mathbf{a}}(\omega)\right)(\omega \in \Omega)$. Therefore, the monotone convergence theorem gives

$$
\int_{\Omega} f\left(b^{1 / n}(\omega)\right) d \mu(\omega) \rightarrow \int_{\Omega} f\left(p^{\mathbf{a}}(\omega)\right) d \mu(\omega) \quad(\mu \in \mathfrak{M}) .
$$

This shows that for any $\mu \in \mathfrak{M}$, the sequence $\left\{\Phi_{\mu}\left(\kappa_{A}^{\mathbf{a}}(b)\right)^{1 / n}\right\}_{n \in \mathbb{N}}$ will weak- ${ }^{*}$-converge to $\Phi_{\mu}\left(p^{\mathbf{a}}\right)$. Since the isomorphism in (5.1) is normal, we see from (5.3) that $\left\{\kappa_{A}(b)^{1 / n}\right\}_{n \in \mathbb{N}} \sigma^{*}-$ converges to $\Phi\left(p^{\mathbf{a}}\right)$. Similarly, $q=\Phi\left(q^{\mathbf{a}}\right)$. Thus, we have $\Phi(u) \Phi(u)^{*}=p$ and $\Phi(u)^{*} \Phi(u)=q$ as required.

Remark 5.5. We are grateful to the referee for communicating to us a shorter proof for the above proposition using a result in [18]. With that proof, one can bypass Lemma 5.1(b), whose argument is lengthy and complicated (note that the case when $n<\infty$ of Lemma 5.1(b) is still needed for Theorem 5.3). However, since we think that the fact stated in Lemma 5.1(b) could be interesting to some other people, we decided to keep our proof. Nevertheless, for the benefit of the reader, we include the outline of the proof from the referee in the following.

Since $A$ is separable, there exist $a, b \in A_{+}$such that $p=\sigma^{*}-\lim _{n} \kappa_{A}(a)^{1 / n}$ and $q=\sigma^{*}$ $\lim _{n} \kappa_{A}(b)^{1 / n}$, respectively. Suppose that $\operatorname{rank} p^{\mathbf{a}}(\omega)=\operatorname{rank} q^{\mathbf{a}}(\omega)(\omega \in \Omega)$. For any lower 
semi-continuous tracial weight $\tau$ on $A$, there is a finite Radon measure $\mu$ on $\Omega$ such that $\tau$ is given by the integration with respect to $\mu$ composing with the trace on $\mathcal{B}\left(\ell^{2}\right)$. If $\tilde{\tau}$ is the normal extension of $\tau$ to $A^{* *}$, then $d_{\tau}(a)=\tilde{\tau}\left(p^{\mathbf{a}}\right)=\int_{\Omega} \operatorname{rank} p^{\mathbf{a}}(\omega) d \mu(\omega)$ and a similar relation holds for $d_{\tau}(b)$. Thus, the above assumption implies that $d_{\tau}(a)=d_{\tau}(b)$ and $[18$, Theorem 5.8] gives $p \sim_{\mathrm{Mv}} q$ as required.

Theorem 5.6. Let $\Theta: \operatorname{Cu}\left(C_{0}(\Omega)\right) \rightarrow \operatorname{Lsc}\left(\Omega, \overline{\mathbb{N}}_{0}\right)$ be the map induced by (5.4).

(a) $\Theta$ is surjective.

(b) $\Theta$ is injective if and only if $\sim_{\mathrm{Mv}}$ coincides with $\sim_{\mathrm{Cu}}$ on $\mathrm{OP}\left(C_{0}(\Omega) \otimes \mathcal{K}\right)$.

Proof: (a) We first fix an orthonormal basis $\left\{\varsigma^{(k)}\right\}_{k \in \mathbb{N}}$ for $\ell^{2}$ and consider elements in $\mathcal{B}$ as infinite matrices. Let $e_{0}:=0, e_{\infty}:=\mathrm{id}_{\ell^{2}}$, and $e_{n}:=\sum_{k=1}^{n} \theta_{\varsigma^{(k)}, \varsigma^{(k)}}(n \in \mathbb{N})$. Suppose that $f \in \operatorname{Lsc}\left(\Omega, \overline{\mathbb{N}}_{0}\right)$. For any $\omega \in \Omega$, we set

$$
h_{f, \varsigma}(\omega)=e_{n} \text { whenever } f(\omega)=n .
$$

Since for any $\omega_{0} \in \Omega$, the map $\omega \mapsto \min \left\{f(\omega), f\left(\omega_{0}\right)\right\}$ is continuous at $\omega_{0}$, we see that $h_{f, \varsigma} \in \mathrm{OP}^{\mathbf{a}}\left(C_{0}(\Omega) \otimes \mathcal{K}\right.$ ) (by Proposition 4.1) and the surjectivity is obtained.

(b) Notice that $\hat{\Theta}(p)=\hat{\Theta}(q)$ if and only if $p^{\mathbf{a}}$ is Murray-von Neumann equivalent to $q^{\mathbf{a}}$ in $\ell^{\infty}(\Omega ; \mathcal{B})$, which in turn is equivalent to $p \sim_{\mathrm{Mv}} q$ due to Proposition 5.4. This gives the conclusion.

Corollary 5.7. Let

$\mathrm{OP}_{\mathrm{s}}\left(C_{0}(\Omega) \otimes \mathcal{K}\right):=\left\{p \in \mathrm{OP}\left(C_{0}(\Omega) \otimes \mathcal{K}\right): \Gamma_{\infty}^{p}=\emptyset\right.$ and $\Gamma_{n}^{p} \in \mathcal{O}(\Omega)$ for every $\left.n \in \mathbb{N}\right\}$.

(a) Suppose that $\Theta: \operatorname{Cu}\left(C_{0}(\Omega)\right) \rightarrow \operatorname{Lsc}\left(\Omega, \overline{\mathbb{N}}_{0}\right)$ is injective. Let $U \in \mathcal{O}(\Omega), n \in \mathbb{N}$ and $h: U \rightarrow \mathcal{P}_{n}$ be a norm-continuous map. If $V \in \mathcal{O}(\Omega)$ such that $V \Subset U$, there is a normcontinuous map $\varphi: V \rightarrow \mathcal{J}_{n}$ satisfying $\varphi \varphi^{*}=\left.h\right|_{V}$, i.e., $\varphi(v) \varphi(v)^{*}=h(v), \forall v \in V$.

(b) $\sim_{\mathrm{PZ}}$ coincides with $\sim_{\mathrm{Mv}}$ on $\operatorname{OP}_{\mathrm{s}}\left(C_{0}(\Omega) \otimes \mathcal{K}\right)$ if and only if for any $n \in \mathbb{N}, U \in \mathcal{O}(\Omega)$ and norm-continuous map $h: U \rightarrow \mathcal{P}_{n}$, there is a norm-continuous map $\psi: U \rightarrow \mathcal{J}_{n}$ satisfying $\psi \psi^{*}=h$.

Proof: Let $\varsigma^{(k)}$ and $e_{k}$ be as in the proof of Theorem $5.6\left(k \in \mathbb{N}_{0}\right)$.

(a) Let us first extend $h$ to a function from $\Omega$ to $\operatorname{Proj}(\mathcal{B})$ by setting $h(\Omega \backslash U)=\{0\}$. Then Proposition 4.1 produces a unique element $p \in \operatorname{OP}(A)$ with $h=p^{\mathbf{a}}$. Similarly, if we set $g \equiv e_{n}$ on $U$ and $g \equiv 0$ on $\Omega \backslash U$, then $g=q^{\mathbf{a}}$ for a unique $q \in \operatorname{OP}(A)$. The same argument also tells us that there exist $r, s \in \mathrm{OP}(A)$ with $r^{\mathbf{a}}=\chi_{V} \cdot h$ and $s^{\mathbf{a}}=\chi_{V} \cdot g$. By multiplying 
$h$ and $g$ with a continuous function from $\Omega$ to $[0,1]$ that takes the value 1 on $\bar{V}$ and vanishes outside $U$, we obtain $r \Subset p$ and $s \Subset q$ (see the discussion following Proposition 3.1). The injectivity of $\Theta$ and Theorem 5.6(b) tell us that $p \sim_{\mathrm{Cu}} q$. Thus, $r \sim_{\mathrm{PZ}} s_{1}$ for an open projection $s_{1} \Subset q$. As $\operatorname{rank} r^{\mathbf{a}}=\operatorname{rank} s_{1}^{\mathbf{a}}$, we deduce that $s_{1}=s$. Let $u \in \ell^{\infty}(\Omega ; \mathcal{B}) \cong z_{\mathbf{a}} A^{* *}$ be an element satisfying the condition in Proposition 3.1(b) for $r \sim_{\mathrm{PZ}} s$ such that $u u^{*}=r$ and $u^{*} u=s$. Then $\left.u u^{*}\right|_{V}=\left.h\right|_{V}$ and $\left.u^{*} u\right|_{V}=\left.g\right|_{V}$. Define $\varphi: V \rightarrow \mathcal{J}_{n}$ by

$$
\varphi(\omega)\left(\lambda_{1}, \ldots, \lambda_{n}\right):=u(\omega)\left(\sum_{i=1}^{n} \lambda_{i} \varsigma^{(i)}\right) \quad\left(\omega \in V ; \lambda_{1}, \ldots, \lambda_{n} \in \mathbb{C}\right)
$$

(notice that $u^{*}(\omega) u(\omega)=e_{n}$ ). Now, pick any $\omega_{0} \in V$ and any open neighbourhood $W$ of $\omega_{0}$ with $W \Subset V$. By multiplying $g$ with a continuous function from $\Omega$ to $[0,1]$ that takes the value 1 on $\bar{W}$ and vanishes outside $V$, we obtain $b \in \operatorname{her}(s)_{+}$such that $\left.\kappa_{A}^{\mathrm{a}}(b)\right|_{W}=\left.g\right|_{W}$. As $u \kappa_{A}^{\mathbf{a}}(b) \in \kappa_{A}^{\mathbf{a}}(A)$ and $\left.u\right|_{W}=\left.u \kappa_{A}^{\mathbf{a}}(b)\right|_{W}$, one knows that $\left.u\right|_{W}$ is norm-continuous at $\omega_{0}$. Hence, $\varphi$ is norm-continuous at $\omega_{0}$.

(b) The argument of the "only if part" is similar to, but easier than, that of part (a).

For the "if part", let us suppose that $p, q \in \mathrm{OP}_{\mathrm{s}}(A)$ with $p \sim_{\mathrm{Mv}} q$. Then $\Gamma_{n}^{p}=\Gamma_{n}^{q}\left(n \in \mathbb{N}_{0}\right)$. Consider $r \in \mathrm{OP}_{\mathrm{s}}(A)$ with $r^{\mathbf{a}}(\omega)=e_{n}$ for all $\omega \in \Gamma_{n}^{p}$ and $n \in \mathbb{N}_{0}$ (whose existence is ensured by Proposition 4.1). If $n \in \mathbb{N}$, we define $h_{n}:=\left.p^{\mathrm{a}}\right|_{\Gamma_{n}^{p}}$. By Corollary 4.3(b), $h_{n}$ is norm-continuous and the hypothesis produces a norm-continuous map $\psi_{n}: \Gamma_{n}^{p} \rightarrow \mathcal{J}_{n}$ with $\psi_{n} \psi_{n}^{*}=h_{n}$. Now, define $u \in \ell^{\infty}(\Omega ; \mathcal{B})$ by

$$
u(\omega)\left(\sum_{k=1}^{\infty} \lambda_{k} \varsigma^{(k)}\right):= \begin{cases}\psi_{n}(\omega)\left(\lambda_{1}, \ldots, \lambda_{n}\right) & \text { if } \omega \in \Gamma_{n}^{p} \\ 0 & \text { if } \omega \in \Gamma_{0}^{p}\end{cases}
$$

Then $\left.u\right|_{\Gamma_{n}^{p}}$ is norm-continuous for every $n \in \mathbb{N}_{0}$, and we have $u u^{*}=p^{\mathbf{a}}$ as well as $u^{*} u=r^{\mathbf{a}}$. Suppose that $f \in \operatorname{her}(p)$. Note that $\left\{\Gamma_{n}^{p}\right\}_{n \in \mathbb{N}}$ is a disjoint sequence in $\mathcal{O}(\Omega)$ and

$$
\left\|f\left(\omega_{j}\right)\right\| \rightarrow\left\|f\left(\omega_{0}\right)\right\|=\left\|p^{\mathbf{a}}\left(\omega_{0}\right) f\left(\omega_{0}\right)\right\|=0
$$

whenever $\left\{\omega_{j}\right\}_{j \in J}$ is a net in $\Omega$ converging to $\omega_{0} \in \Gamma_{0}^{p}$. It follows $u^{*} \kappa_{A}^{\mathbf{a}}(f) \in \kappa_{A}^{\mathbf{a}}(A)$. Similarly, we have $u \kappa_{A}^{\mathrm{a}}(\operatorname{her}(r)) \subseteq \kappa_{A}^{\mathrm{a}}(A)$, which implies that $p \sim_{\mathrm{PZ}} r$, because of Proposition 3.1(b). A similar argument also shows that $q \sim_{\mathrm{PZ}} r$.

Corollary 5.8. If $\operatorname{dim} \Omega \leq 1$ and $A=C_{0}(\Omega) \otimes \mathcal{K}$, then $\sim_{\mathrm{Mv}}, \sim_{\mathrm{PZ}}, \sim_{\mathrm{sp}}$ and $\sim_{\mathrm{Cu}}$ coincide with each other on $\mathrm{OP}(A)$.

Proof: It suffices to show that $\sim_{\mathrm{Mv}}$ coincides with $\sim_{\mathrm{PZ}}$ on $\mathrm{OP}(A)$. Note that since $\operatorname{dim} \Omega \leq$ 1, the topological stable rank of $C_{0}(\Omega)$ is 1 , and so is $A$ (by [17, Theorem 6.4]). Thus, [18, $\left.\S 6.2\right]$ tells us that $\sim_{\mathrm{Cu}}$ coincides with $\sim_{\mathrm{PZ}}$ on $\mathrm{OP}(A)$. Now, the result follows from $[3$, Theorem 3.4] and Theorem 5.6(b) (note that although the result in [3] was stated for the case of 
$\operatorname{dim} \Omega=1$, it actually holds for $\operatorname{dim} \Omega=0$ as well, by using exactly the same argument).

The fact that $[3$, Theorem 3.4] holds for the case when $\operatorname{dim} \Omega=0$ was communicated to us by the referee. Our original proof involves a separate lengthy argument for this case.

Remark 5.9. (a) The proof of Theorem 5.3 tells us that the conclusion of Corollary 5.7(a) does not hold when $\Omega=\mathbb{C P}^{1}$.

(b) Suppose that $\sim_{\mathrm{PZ}}$ coincides with $\sim_{\mathrm{Mv}}$ on $\mathrm{OP}_{\mathrm{s}}\left(C_{0}(\Omega) \otimes \mathcal{K}\right)$. For any $p \in \mathrm{OP}\left(C_{0}(\Omega) \otimes \mathcal{K}\right)$, we obtain from Corollary 5.7(b) and Corollary 4.3(c) a norm continuous map $\psi: \Omega_{0} \rightarrow \bigcup_{n \in \mathbb{N}_{0}} \mathcal{J}_{n}$ such that $\psi \psi^{*}=\left.p^{\mathbf{a}}\right|_{\Omega_{0}}$. Notice also that if $\Gamma_{\infty}^{p}=\emptyset$, then Corollary $4.3(\mathrm{~d})$ says that $\Omega_{0}$ is dense in $\Omega$.

By [21, Theorems 1.1 and 1.3] and Theorem 5.6, we have the following result.

Corollary 5.10. The equivalence relations $\sim_{\mathrm{Cu}}$ and $\sim_{\mathrm{Mv}}$ coincide on $\mathrm{OP}\left(C_{0}(\Omega) \otimes \mathcal{K}\right)$ if and only if $\operatorname{dim} \Omega \leq 2$ and the Cěch cohomology $\check{H}^{2}(K ; \mathbb{Z})$ vanishes for any compact subset $K \subseteq \Omega$.

\section{ACKNOWLEDGMENT}

This work was initialled during a visit of the first named author to the National Sun YatSen University in November, 2012. He would like to thank the colleagues in the Department of Applied Mathematics there for their hospitality.

The authors would like to thank L. G. Brown for pointing out $[4, \S 5 . \mathrm{G}]$ to them, thank L. Robert for pointing out [22] to them, and thank H. Lin for some helpful discussions.

They would also like to thank the referee for careful reading of the paper and for several helpful comments. In particular, they would like to thank the referee for informing them of a shorter proof for Proposition 5.2 using [18, Theorem 5.8], as well as the fact that [3, Theorem 3.4] actually holds for $\operatorname{dim} \Omega \leq 1$, with which the authors shortened the argument of Corollary 5.8.

\section{REFERENCES}

[1] C. A. Akemann, The General Stone-Weierstrass Problem, J. Funct. Anal. 4 (1969), 277-294.

[2] C. A. Akemann, A Gelfand representation theory for $C^{*}$-algebras, Pacific J. Math. 39 (1971), 1-11.

[3] R. Antoine, F. Perera and L. Santiago, Pullbacks, $C(X)$-algebras, and their Cuntz semigroup, J. Funct. Anal. 260 (2011), 2844-2880.

[4] L. G. Brown, Semicontinuity and multipliers of C*-algebras, Can. J. Math. XL (1988), 865-988. 
[5] L. G. Brown and N. C. Wong, On $C^{*}$-algebras cut down by closed projections: characterizing elements via the extreme boundary, Taiwanese J. Math. 5 (2001), 433-441.

[6] J. P. Burgess, A selection theorem for group actions, Pacific J. Math. 80 (1979), 333-336.

[7] J. Dixmier, Dual et quasi-dual d'une algebre de Banach involutive, Trans. Amer. Math. Soc. 104 (1962), $278-283$

[8] E. G. Effros, Transformation groups and $C^{*}$-algebras, Ann. of Math. 81 (1965), 38-55.

[9] R. V. Kadison and J. R. Ringrose, Fundamentals of the theory of operator algebras Vol. II: Advanced theory, Pure and Applied Mathematics 100, Academic Press (1986).

[10] H. Lin, Equivalent open projections and corresponding hereditary $C^{*}$-subalgebras, J. London Math. Soc. (2), 41 (1990), 295-301.

[11] H. Lin, An introduction to the classification of amenable $C^{*}$-algebras, World Scientific Publishing Co., Inc., River Edge, NJ (2001).

[12] H. Lin, Cuntz semigroups of $C^{*}$-algebras of stable rank one and projective Hilbert modules, preprint (arXiv:1001.4558).

[13] G. W. Mackey, Borel structures in groups and their duals, Trans. Amer. Math. Soc. 85 (1957), 134-165.

[14] F. J. Murray, The rings of operators papers, in The legacy of John von Neumann (Hempstead, NY, 1988), 57-60, Proc. Sympos. Pure Math. 50, Amer. Math. Soc., Providence, R.I. (1990).

[15] F. J. Murray and J. Von Neumann, On rings of operators, Ann. of Math. (2), 37 (1936), 116-229.

[16] C. K. Ng and N. C. Wong, A Murray-von Neumann type classification of $C^{*}$-algebras, preprint (arXiv:1112.1455).

[17] V. Nistor, Stable range for tensor products of extensions of $\mathcal{K}$ by $C(X)$, J. Oper. Theory 16 (1986), 387-396.

[18] E. Ortega, M. Rørdam and H. Thiel, The Cuntz semigroup and comparison of open projections, J. Funct. Anal. 260 (2011), 3474-3493.

[19] C. Peligrad and L. Zsidó, Open projections of $C^{*}$-algebras: Comparison and Regularity, Operator Theoretical Methods, 17th Int. Conf. on Operator Theory, Timisoara (Romania), June 23-26, 1998, Theta Found. Bucharest (2000), 285-300

[20] G. K. Pedersen, $C^{*}$-algebras and their automorphism groups, Academic Press (1979).

[21] L. Robert, The Cuntz semigroup of some spaces of dimension at most 2, C. R. Math. Acad. Sci. Soc. R. Can. 35 (2013), 22-32.

[22] L. Robert and A. Tikuisis, Hilbert $C^{*}$-modules over a commutative $C^{*}$-algebra, Proc. Lond. Math. Soc. 102 (2011), 229-256.

(Chi-Keung Ng) Chern Institute of Mathematics and LPMC, Nankai University, Tianjin 300071, CHINA.

E-mail address: ckng@nankai.edu.cn

(Ngai-Ching Wong) Department of Applied Mathematics, National Sun Yat-Sen University, KAOHSIUNG, 80424, TAIWAN.

E-mail address: wong@math.nsysu.edu.tw 